\title{
Effectiveness of Screening and Treatment Approaches for Schistosomiasis and Strongyloidiasis in Newly-Arrived Migrants from Endemic Countries in the EU/EEA: A Systematic Review
}

Eric N. Agbata ${ }^{1,2, *}$, Rachael L. Morton ${ }^{3} \oplus$, Zeno Bisoffi ${ }^{4,5}$, Emmanuel Bottieau ${ }^{6}$, Christina Greenaway ${ }^{7}$, Beverley-A. Biggs ${ }^{8,9}$, Nadia Montero ${ }^{10}{ }^{\circledR}$, Anh Tran ${ }^{3}$, Nick Rowbotham ${ }^{3}$, Ingrid Arevalo-Rodriguez $\left.{ }^{10,11}{ }^{(}\right)$, Daniel T. Myran ${ }^{12}$, Teymur Noori ${ }^{13}\left(\mathbb{C}^{\circ}\right.$, Pablo Alonso-Coello ${ }^{14}$, Kevin Pottie ${ }^{15}$ and Ana Requena-Méndez ${ }^{16}$

1 Faculty of Health Science, University of Roehampton London, London SW15 5PU, UK

2 Department of Paediatrics, Obstetrics, Gynaecology and Preventive Medicine, Universitat Autònoma de Barcelona, Bellaterra, 08193 Barcelona, Spain

3 NHMRC Clinical Trials Centre, University of Sydney, Camperdown, NSW 2050, Australia;

Rachael.morton@ctc.usyd.edu.au (R.L.M.); anh.tran@ctc.usyd.edu.au (A.T.); rowbothamn@gmail.com (N.R.)

4 Centre for Tropical Diseases (CTD), IRCCS Sacro Cuore Don Calabria Negrar, Negrar, 37024 Verona, Italy; zeno.bisoffi@sacrocuore.it

5 Department of Diagnostics and Public Health, University of Verona, 37134 Verona, Italy

6 Department of Clinical Sciences, Institute of Tropical Medicine, 155 Nationalestraat, 2000 Antwerp, Belgium; EBottieau@itg.be

7 Division of Infectious Diseases and Clinical Epidemiology, Sir Mortimer B. Davis-Jewish General Hospital, McGill University, Montreal, QC H3A 0G4, Canada; ca.greenaway@mcgill.ca

8 Department of Medicine at the Doherty Institute, University of Melbourne, Parkville, VIC 3010, Australia; babiggs@unimelb.edu.au

9 Victorian Infectious Diseases Service, The Royal Melbourne Hospital RMH, Parkville, VIC 3050, Australia

10 Centro de Investigación en Salud Pública y Epidemiología Clínica (CISPEC), Facultad de Ciencias de la Salud Eugenio Espejo, Universidad Tecnológica Equinoccial, Quito 170509, Ecuador; nadiamonteromd@gmail.com (N.M.); inarev7@yahoo.com (I.A.-R.)

11 Clinical Biostatistics Unit, Hospital Universitario Ramon y Cajal (IRYCIS); CIBER Epidemiology and Public Health (CIBERESP), 28034 Madrid, Spain

12 Bruyere Research Institute, University of Ottawa, Ottawa, ON K1N 6N5, Canada; daniel.myran@gmail.com

13 European Centre for Disease Prevention and Control, Gustav III: s Boulevard 40, 16973 Solna, Sweden; Teymur.Noori@ecdc.europa.eu

14 Iberoamerican Cochrane Center, Biomedical Research Institute Sant Pau (IIB Sant Pau-CIBERESP), 08025 Barcelona, Spain; PAlonso@santpau.cat

15 Centre for Global Health Institute of Population Health, University of Ottawa, Ottawa, ON K1N 6N5, Canada; kpottie@uottawa.ca

16 ISGlobal, Barcelona Institute for Global Health (ISGlobal-CRESIB, Hospital Clínic-University of Barcelona), E-08036 Barcelona, Spain; ana.requena@isglobal.org

* Correspondence: eric.agbata@roehampton-online.ac.uk

Received: 15 September 2018; Accepted: 17 December 2018; Published: 20 December 2018

\begin{abstract}
We aimed to evaluate the evidence on screening and treatment for two parasitic infections—schistosomiasis and strongyloidiasis-among migrants from endemic countries arriving in the European Union and European Economic Area (EU/EEA). We conducted a systematic search of multiple databases to identify systematic reviews and meta-analyses published between 1 January 1993 and 30 May 2016 presenting evidence on diagnostic and treatment efficacy and cost-effectiveness. We conducted additional systematic search for individual studies published between 2010 and 2017. We assessed the methodological quality of reviews and studies using the
\end{abstract}


AMSTAR, Newcastle-Ottawa Scale and QUADAS-II tools. Study synthesis and assessment of the certainty of the evidence was performed using GRADE (Grading of Recommendations Assessment, Development and Evaluation) approach. We included 28 systematic reviews and individual studies in this review. The GRADE certainty of evidence was low for the effectiveness of screening techniques and moderate to high for treatment efficacy. Antibody-detecting serological tests are the most effective screening tests for detection of both schistosomiasis and strongyloidiasis in low-endemicity settings, because they have higher sensitivity than conventional parasitological methods. Short courses of praziquantel and ivermectin were safe and highly effective and cost-effective in treating schistosomiasis and strongyloidiasis, respectively. Economic modelling suggests presumptive single-dose treatment of strongyloidiasis with ivermectin for all migrants is likely cost-effective, but feasibility of this strategy has yet to be demonstrated in clinical studies. The evidence supports screening and treatment for schistosomiasis and strongyloidiasis in migrants from endemic countries, to reduce morbidity and mortality.

Keywords: migrant populations; schistosomiasis/schistosoma; strongyloidiasis/strongyloides; screening/diagnosis; treatment; public health; GRADE

\section{Introduction}

The public health importance of schistosomiasis and strongyloidiasis has increased in non-endemic regions as a result of growing global migration [1,2]. Schistosomiasis is caused by species of the trematode Schistosoma spp. Sc. mansoni is the most prevalent in Africa, the Americas, the Middle East and the West Indies, followed by Sc. haematobium in Africa and the Middle East and Sc. japonicum in east and south-east Asia [3]. Sub-Saharan African countries account for $90 \%$ of reported cases globally [3]. Prevalence rates of $10-50 \%$ for Sc. haematobium infections have been reported in some countries in sub-Saharan Africa and the Middle East [4], and prevalence rates of $1-40 \%$ have been reported for Sc. mansoni in sub-Saharan Africa and South America and for Sc. japonicum in Indonesia, parts of China and south-east Asia [5].

Strongyloidiasis is caused by the nematode Strongyloides stercoralis and, although it generally occurs in sub-tropical and tropical countries, it can be present in temperate countries where conditions are favourable [6]. The global burden of both diseases has been underestimated because of the poor sensitivity of diagnostic methods used in low-resource settings [6], but recent estimates indicate that around 370 million people are infected with St. stercoralis [7] and more than 200 million are infected with schistosomiasis causing a loss of more than 1.53 million disability-adjusted life years (DALYs) $[4,5,8,9]$.

Few studies have assessed the prevalence schistosomiasis in European countries, but recent data show rates above $17 \%$ in migrants from sub-Saharan Africa [10]; prevalence of strongyloidiasis among refugee populations originating from south-east Asia and Africa was reported to be between $0.8 \%$ and $4.3 \%$ using microscopy; higher rates of between $9 \%$ and $77 \%$ using antibody detection assays were reported among refugees from south-east Asia [11]. Prevalence rates of 3.3\%, 4.2\% and 5.6\% were reported in Italy, Spain and France, respectively, mainly in migrant populations or expatriates, without specifying diagnostic methods [6].

From all parasitic infections that may be highly prevalent among migrants, schistosomiasis and strongyloidiasis have several characteristics which support the rationale for screening based on the classical principles of Wilson and Jungner [12]. First, both infections are of particular importance, besides being as highly prevalent as other parasitic infections, they can cause long-term complications and severe consequences. Schistosomiasis is associated with chronic urogenital, hepato-intestinal and central nervous system complications [9,13-15]. St. stercoralis can cause disseminated infections or hyper infections with fatal outcomes in immunosuppressed patients (e.g., transplant recipients, 
those on corticosteroid therapy, with malignancies or co-infections with human T-cell lymphotropic virus-1 (HTLV-1)) [16]. In addition, there is a potential risk of transmission in the EU/EEA, either through organ transplantation in the case of strongyloidiasis [17] or through a favourable environment for the intermediate host, as in recent autochthonous cases of urinary schistosomiasis in Corsica, France which is not the case from many other parasitic infections [11,18]. Second, most infections are asymptomatic $[13,19,20]$ and those infected are either unaware of their infection [19] or have very mild unspecific symptoms [3]. Third, both are chronic infections if untreated [19]. Schistosomiasis can remain as a sub-clinical infection for many years [3], and St. stercoralis replicates indefinitely inside the human host, causing lifelong infection if untreated [19].

Fourth, screening could be based on a simple and widely accessible technology, including commercially available serological test with a reasonable cost. In this sense, diagnosis of both infections based on microscopy has high specificity but low sensitivity [19,21,22]. Antibody-detecting serological tests offer higher sensitivity, at the expense of specificity, and have been shown to be useful in countries with low endemicity $[19,22,23]$. Finally, treatments for both infections are universally accepted with a high efficacy rate and low rate of adverse events. Praziquantel and ivermectin are the drugs of choice for treating schistosomiasis and strongyloidiasis, respectively $[7,13]$.

In the last ten years, there has been a significant increase in migration patterns to the EU/EEA with some fluctuations in the volume and type of migration from year to year [24,25]. In 2017, migrants, here defined as being born abroad, made up $11 \%$ of this population, with $4 \%$ being born in another EU/EEA country and 7\% originating from outside the EU/EEA [26]. There is an increased number of asylum applications with $56 \%$ of the 2,672,000 asylum decisions being positive between 2015 to 2017 [27]. Half of those denied asylum can be expected to leave, adding 580,000 to the EU/EEA's total number of irregular migrants [28].

There is a notable gap in data collection on the disease burden, public health management, and in the surveillance for imported diseases in migrants arriving from endemic areas to EU/EEA. Geographic differences in disease distribution between global regions, influenced by increasing migration and population mobility from high endemic to non-endemic areas, remains an ongoing challenge to surveillance programmes and hampers the implementation of health policies concerning migrant health screening strategies [29,30].

There have been several systematic reviews addressing how effective are approaches to migrant screening infectious diseases in Europe [31-33], however parasitic infections are not adequately covered. Therefore, given the recent increase in migrants to the EU/EEA from endemic countries, there is a need for public health guidelines on the optimal approach to screening for schistosomiasis and strongyloidiasis [34-36]. In this systematic review, we assessed the effectiveness (and cost-effectiveness) of screening and management of these two parasitic infections in migrant populations.

\section{Methods}

The review was one of six systematic reviews conducted under the auspices of a European Centre for Disease Prevention and Control (ECDC) project to develop guidance on screening for hepatitis C, hepatitis B, HIV, tuberculosis, vaccine-preventable diseases and parasitic infections in newly-arrived migrants to the EU/EEA [37]. The review group followed the Preferred Reporting Items for Systematic Reviews and Meta-Analyses (PRISMA) guidelines for the reporting of this systematic review [38]. The review protocol and methods assembled by a team of methodologists and clinicians with disease expertise was registered in Prospero (CRD42016045798) and published [39].

Our key research question was:

What are the most effective screening and treatment options for schistosomiasis and strongyloidiasis in migrant populations arriving from endemic regions in the EU/EEA?

To address this, we developed a logic model, prioritised outcomes important for the patient, and developed key questions along the evidence pathway (Appendix A). These key questions included: 
(i) What are the best diagnostic tests to detect these infections non-endemic settings?

(ii) How effective are the drugs to treat them and what are the associated adverse events?

(iii) What are the most cost-effective screening and treatment options for schistosomiasis and strongyloidiasis in migrant populations from endemic regions in the EU/EEA?

\subsection{Search Strategy and Selection Criteria}

We searched for systematic reviews and meta-analyses in MEDLINE, Embase-ELSEVIER, the Cumulative Index to Nursing and Allied Health Literature (CINAHL), Epistemonikos, the Database of Abstracts of Reviews of Effects (DARE) and the Cochrane Database of Systematic Reviews (CDSR) for evidence on effectiveness. Our search used a combination of the key terms: 'Immigrant', 'Strongyloides', 'Schistosomiasis', 'endemicity', 'prevalence', 'screening', 'migrant screening', 'mass screening', 'early detection', 'health impact assessment' and 'cost-effectiveness' (Appendix B). The primary inclusion populations were migrants and refugees. We considered as main outcomes: cure, mortality, morbidity, adverse effects, health equity, quality of life and test accuracy measures (sensitivity and specificity). Also, we searched the National Health System (NHS) Economic Evaluation Database, the Health Economic Evaluations Database, the Cost Effectiveness Analysis Registry and Google Scholar for evidence on cost-effectiveness. We also identified any reviews on prevalence of the two infections. We restricted the search to studies published between 1 January 1993 and 30 May 2016. We did not apply language restrictions, and where we identified more than one version of a systematic review, we included the most recent. For the economic evidence, systematic reviews and primary studies of resource use, costs or cost-effectiveness of screening for schistosomiasis or strongyloidiasis with or without treatment were identified using specific search terms including ("costs and cost analysis"; "cost effectiveness analysis"; "costs.tw"; "cost\$.mp"; "cost effective\$.tw"; "cost-benefit analys\$.mp" "health care costs.mp") combined with clinical criteria. We reported all the costs in the local currency of the study setting or country, and in Euros using the Cochrane methods group purchasing power parity currency conversion calculator for the given year [40]. We also searched grey literature for published guidelines and reports on screening and prevention programme from the United States (U.S.) Centers for Disease Control and Prevention, ECDC, Joint United Nations Programme on HIV / AIDS (UNAIDS) and World Health Organization (WHO).

\subsection{Additional Included Studies}

Due to the limited evidence obtained from the initial search, we conducted an updated systematic search of six databases (MEDLINE, Embase-ELSEVIER, CINAHL, CDSR, DARE, Cochrane CENTRAL and Latin American Literature in Health Sciences-LILACS). We included relevant primary studies on diagnostic or screening tools for schistosomiasis (January 2010-February 2017) and strongyloidiasis (January 2012-February 2017). References of included primary studies were searched to identify other relevant studies.

\subsection{Study Selection, Quality Assessment, and Synthesis}

We included systematic reviews and evidence-based review guidelines which addressed each key question. When no systematic review was identified, we used primary studies. Two team members independently screened the titles and abstracts, followed by full-text assessments for eligibility of studies on prevalence, screening and treatment effectiveness, and related key questions (Eric Agbata, Nadia Montero) and of studies on cost-effectiveness (Nick Rowbotham, Rachael Morton). Disagreements were resolved by consensus or the involvement of a third author (AR). We assessed the methodological quality of reviews using AMSTAR [41] or Newcastle-Ottawa Scale [42] for reviews and observational studies respectively. We assessed the methodological quality of included primary studies on diagnostic effectiveness using the Quality Assessment of Diagnostic Accuracy Studies (QUADAS II) tool [43]. Synthesis of the studies and assessment of the certainty of the evidence for systematic reviews and individual studies was performed using GRADE (Grading 
of Recommendations Assessment, Development and Evaluation) methods, including Summary of Findings tables and Evidence to Decision tables [37]. For cost-effectiveness studies, we extracted the following data: economic study design (e.g., cost-utility analysis, Markov model), description of the case base population, the intervention and comparator, the absolute and relative difference in resource use and cost-effectiveness (e.g., incremental net benefit (INB) or incremental cost-effectiveness ratio (ICER).

\section{Results}

The first systematic search yielded, after removal of duplicates, 662 systematic reviews for which we screened titles and abstracts. Of the 26 systematic reviews selected for full-text screening, we included 11 systematic reviews which focused on the efficacy of diagnosis and treatment of schistosomiasis $(n=8)$ and strongyloidiasis $(n=3)$ (Figure 1) [19,44-53]. The updated systematic search for diagnostic testing accuracy studies for schistosomiasis yielded after de-duplication 1961 citations for the screening of titles and abstracts. Of the 30 articles selected for full-text screening, we included seven primary studies (Figure 2) [54-60]. One more primary research was identified later and included [61]. Another systematic search performed for diagnostic testing accuracy evidence for strongyloidiasis yielded 497 records after de-duplication; titles and abstracts were screened, and of the 24 papers selected for full-text screening, we included three primary studies (Figure 3) [62-64]. For the economic evidence, the search strategy yielded 160 studies after de-duplication. We retrieved 20 studies after title and abstract screening, of which six studies (four decision-analytic models for economic evaluation and two costing studies) were finally included-four for strongyloidiasis and two for schistosomiasis (Figure 4) [65-70]. Overall, we included 28 reviews and studies in this systematic review (Tables 1-3).

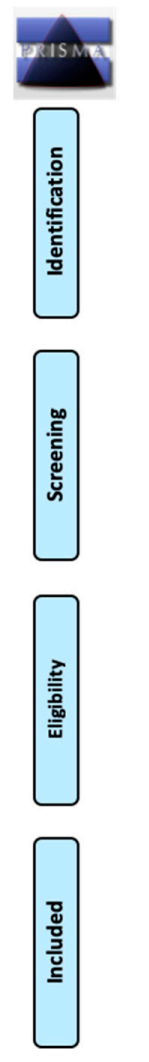

PRISMA 2009 Flow Diagram

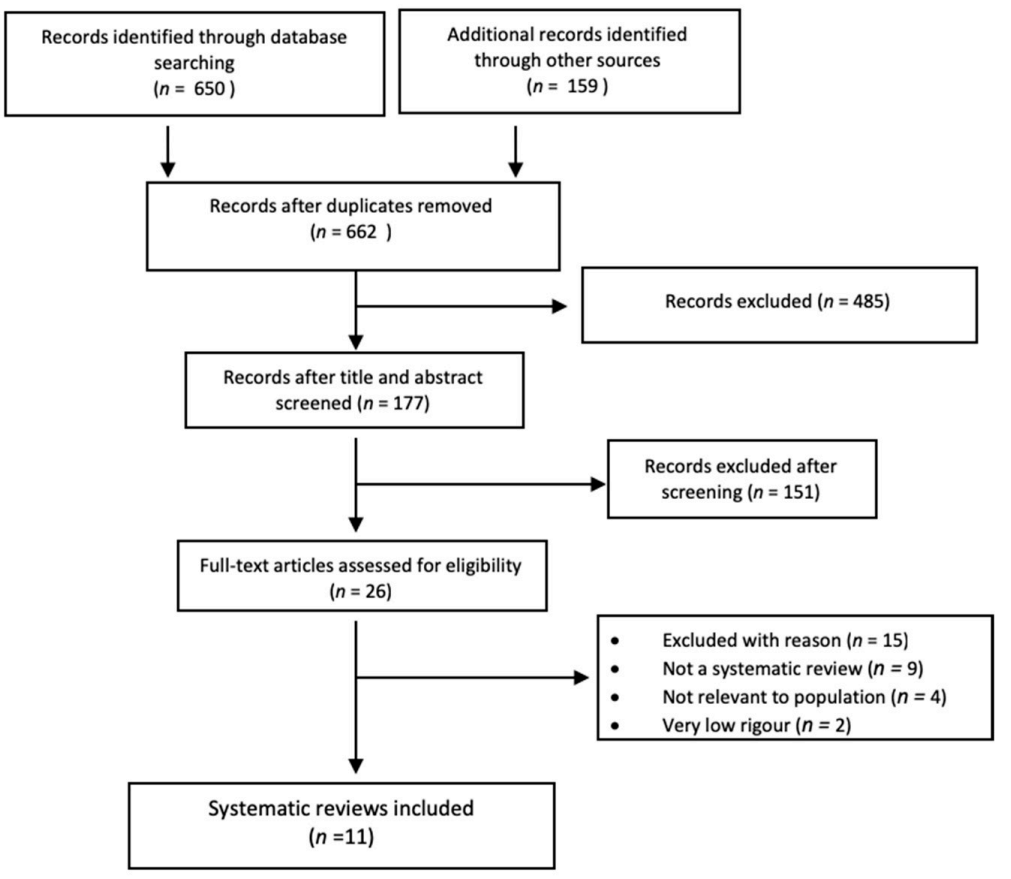

Figure 1. The Preferred Reporting Items for Systematic Reviews and Meta-Analyses (PRISMA) flow diagram for selection of systematic reviews on diagnostic accuracy and treatment efficacy for schistosomiasis and strongyloidiasis, (January 1993-May 2016). 


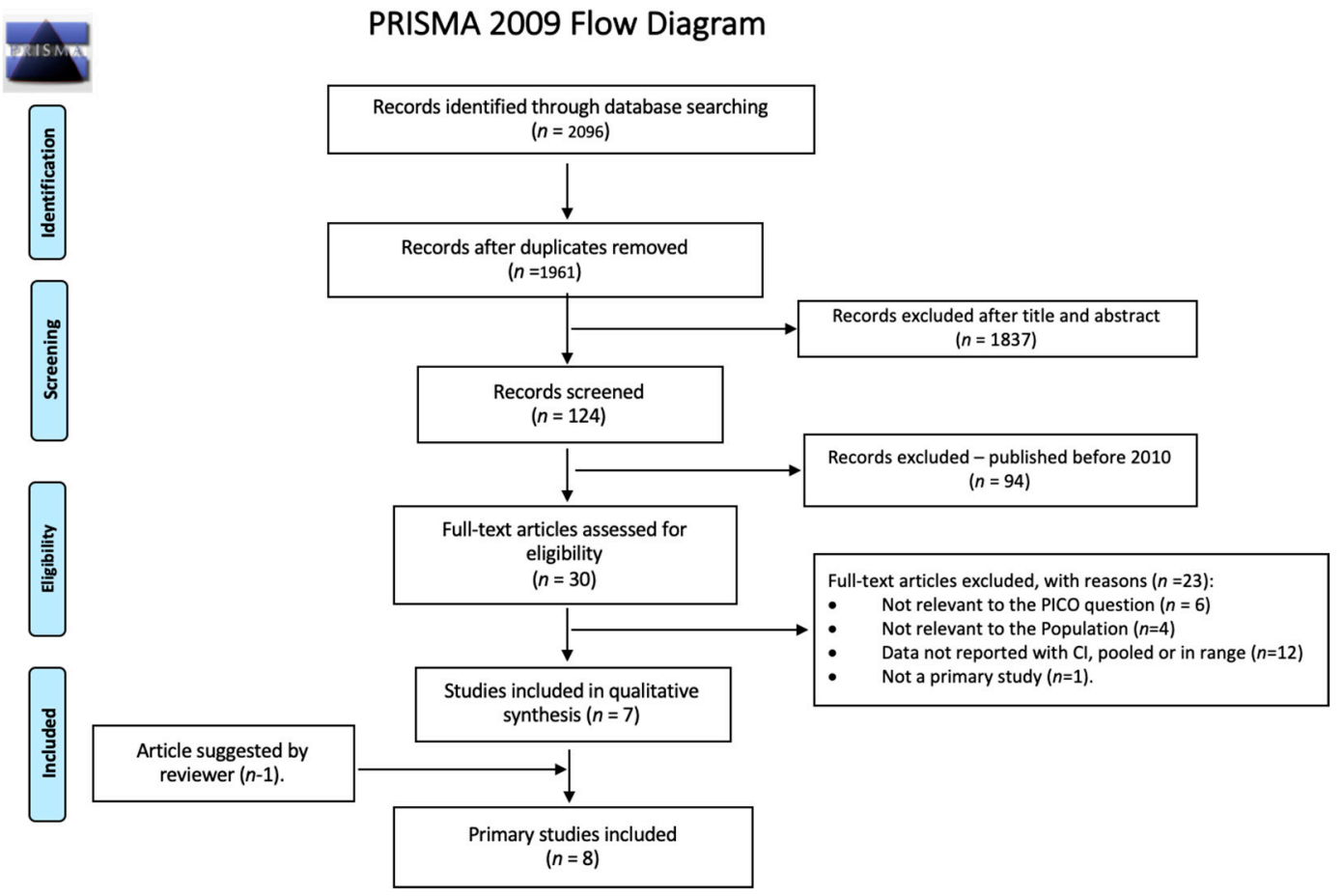

Figure 2. PRISMA flow diagram for selection of primary studies on diagnostic accuracy for schistosomiasis, January 2010-February 2017.

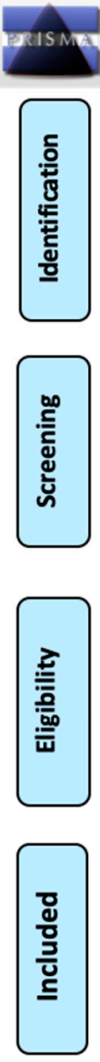

\section{PRISMA 2009 Flow Diagram}

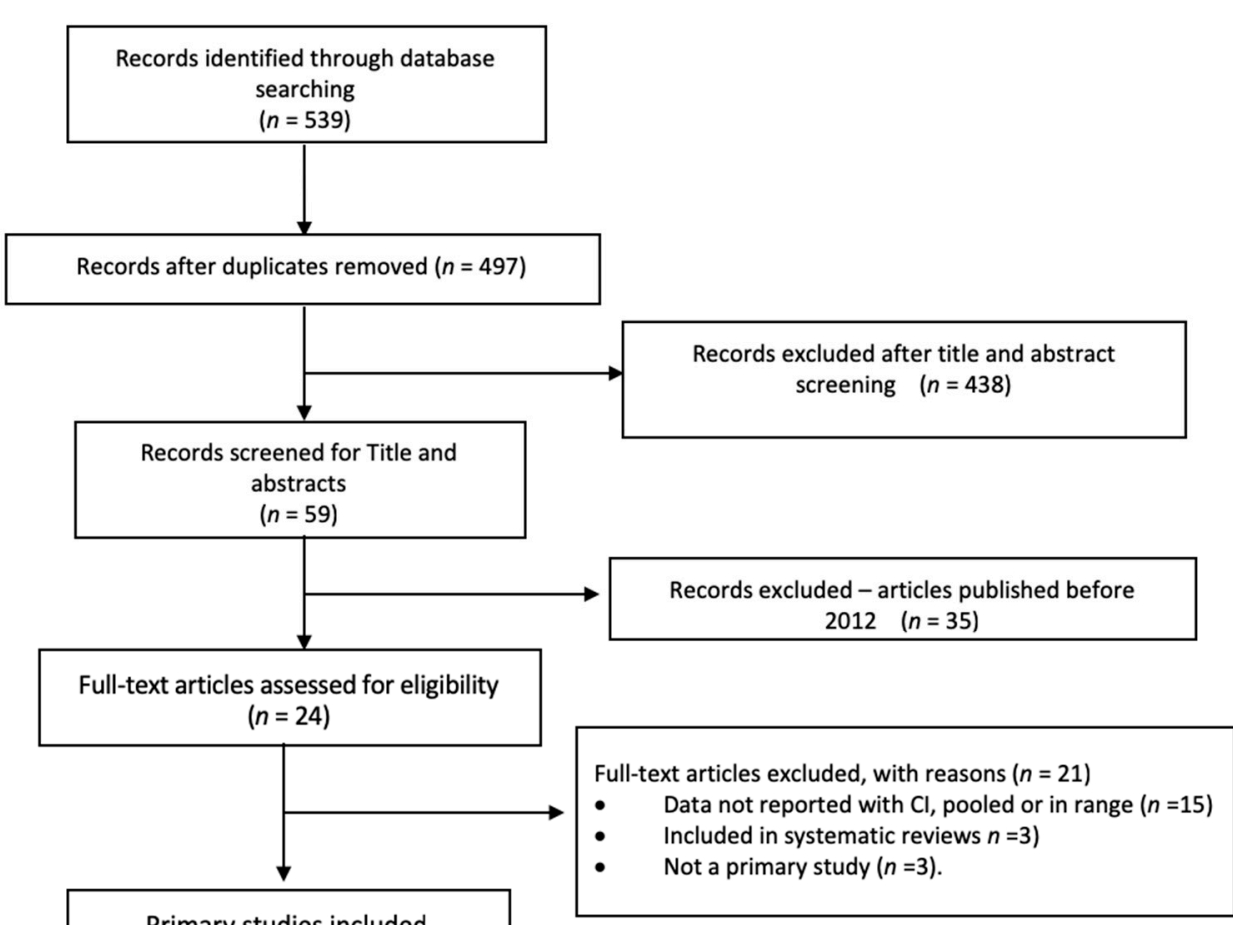

Figure 3. PRISMA flow diagram for selection of primary studies on diagnostic accuracy on strongyloidiasis, (January 2012-February 2017). 


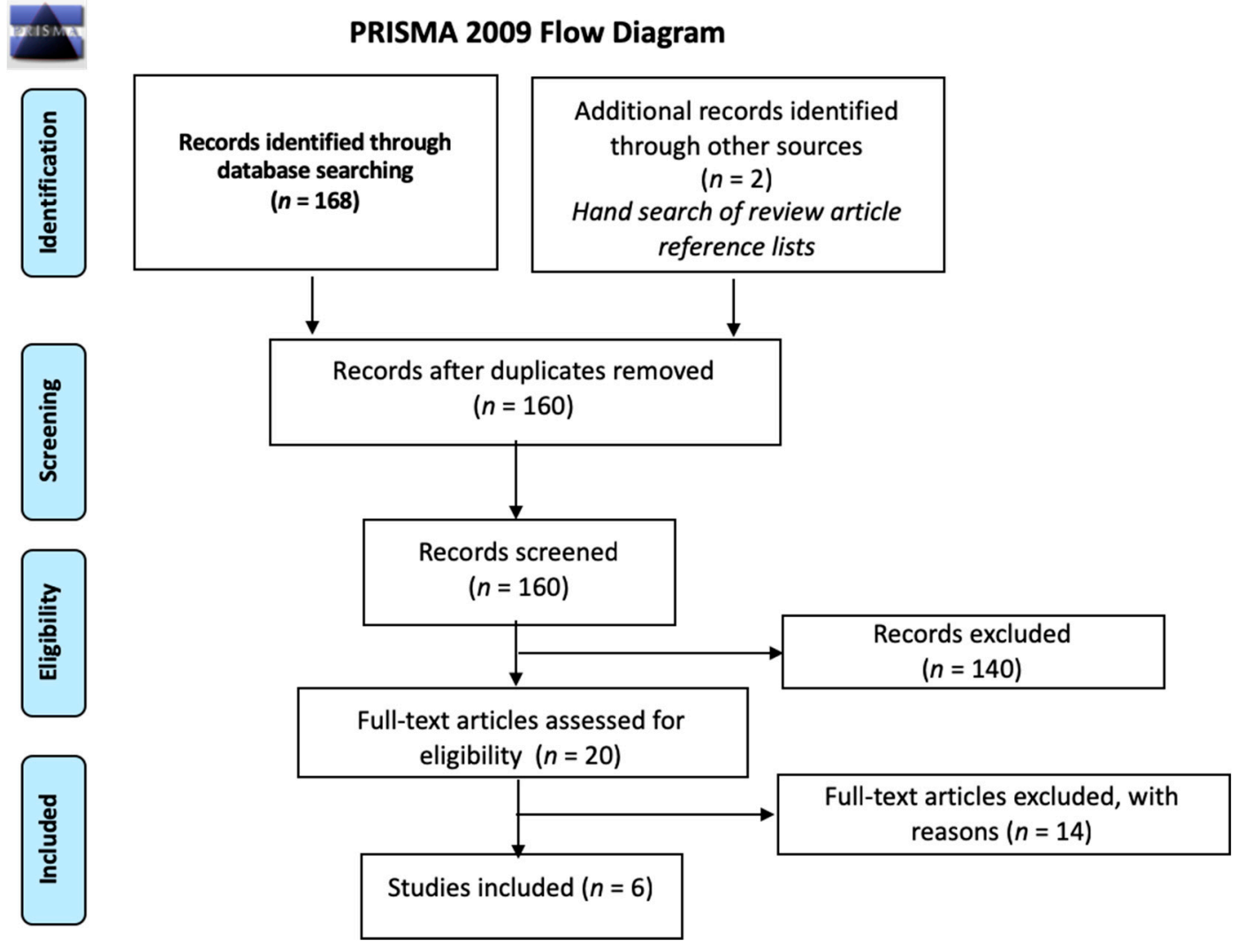

Figure 4. PRISMA flow diagram for selection of cost-effectiveness studies for schistosomiasis and strongyloidiasis, 1993-2016. DARE: Database of Abstracts of Reviews of Effects; NHS EED: National Health Service Economic Evaluation Database; Tufts CEA: Tufts Medical Centre Cost-Effectiveness Analysis Registry. 
Table 1. Characteristics of included studies on diagnostic test effectiveness for schistosomiasis and strongyloidiasis, January $1993-$ February 2017.

\begin{tabular}{|c|c|c|c|c|c|}
\hline Study & Quality & Design & Population & Intervention/Outcomes & Results \\
\hline \multicolumn{6}{|c|}{ Included systematic reviews of diagnostic tests to detect schistosomiasis } \\
\hline $\begin{array}{l}\text { Danso } \\
\text { Appiah et al., } \\
2016[53]\end{array}$ & $\begin{array}{l}\text { AMSTAR: } 11 / 11 \\
\text { GRADE: low to } \\
\text { moderate-quality } \\
\quad \text { evidence }\end{array}$ & $\begin{array}{l}\text { Systematic review and } \\
\text { meta-analysis }\end{array}$ & $\begin{array}{l}\text { Preschool children and infants, } \\
\text { school-aged children or adults } \\
\text { from high-/low-prevalence } \\
\text { locations }\end{array}$ & $\begin{array}{c}\text { Intervention: POC CCA for } \\
\text { Sc. mansoni } \\
\text { Outcomes: detection of } \\
\text { egg-positive } \\
\text { urine-sensitivity/specificity ( } 95 \% \\
\text { CI:) }\end{array}$ & $\begin{array}{c}\text { Sensitivity / specificity }(95 \% \text { CI) } \\
\text { POC CCA (single standard) } 90 \%(84-94) / 56 \% \text { (39-71); } \\
\text { POC CCA (duplicate standard) } 85 \% \text { (80-88)/66\% } \\
\text { (53-76); POC CCA (triplicate standard) } 91 \% \\
\text { (84-95)/56\% (39-72) }\end{array}$ \\
\hline $\begin{array}{l}\text { Yang et al., } \\
2015[50]\end{array}$ & $\begin{array}{l}\text { AMSTAR: } 11 / 11 \\
\text { GRADE: low to } \\
\text { moderate-quality } \\
\text { evidence }\end{array}$ & Meta-analysis & $\begin{array}{c}\text { Patients infected with } \\
\text { schistosomiasis in endemic areas; } \\
\text { mainly school children, Africa } \\
\text { and China } \\
\end{array}$ & $\begin{array}{c}\text { Intervention: questionnaire } \\
\text { screening for Schistosoma species. } \\
\text { Outcomes: sensitivity/specificity } \\
\text { (95\% CI:) }\end{array}$ & $\begin{array}{c}\text { Sensitivity / specificity ( } 95 \% \text { CI:) } \\
\text { Sc. haematobium } 85 \% \text { (84-86)/94\% (94-94); Sc. mansoni } \\
46 \%(45-47) / 81 \% \text { (80-82); Sc. japonicum } 82 \% \\
\text { (79-85)/59\% (57-60) }\end{array}$ \\
\hline $\begin{array}{l}\text { Ochodo et } \\
\text { al., } 2015 \text { [44] }\end{array}$ & $\begin{array}{l}\text { AMSTAR: } 11 / 11 \\
\text { GRADE: very low to } \\
\text { low-quality evidence }\end{array}$ & $\begin{array}{l}\text { Systematic review and } \\
\text { meta-analysis of RCTs }\end{array}$ & $\begin{array}{l}\text { Individuals with active infection } \\
\text { with S. haematobium }\end{array}$ & $\begin{array}{l}\text { Intervention: urine reagent strip } \\
\text { tests; circulating antigen tests in } \\
\text { urine/serum } \\
\text { Outcomes: sensitivity/specificity } \\
\text { ( } 95 \% \text { CI: })\end{array}$ & $\begin{array}{c}\text { Sensitivity /specificity }(95 \% \mathrm{CI}) \\
\text { Sc. haematobuim: microhaematuria } 75 \% \text { (71-79)/87\% } \\
\text { (84-90); proteinuria 61\% (53-68)/82\% (77-88); } \\
\text { leukocyturia } 58 \% \text { ( } 44-71) / 61 \%(34-88) ; \\
\text { Sc. mansoni (CCA test) } 89 \%(86-92) / 55 \%(46-65)\end{array}$ \\
\hline $\begin{array}{c}\text { King and } \\
\text { Bertsch, 2013 } \\
\text { [45] }\end{array}$ & $\begin{array}{l}\text { AMSTAR: } 11 / 11 \\
\text { GRADE: low-quality } \\
\text { evidence }\end{array}$ & $\begin{array}{l}\text { Systematic review and } \\
\text { meta-analysis of surveys }\end{array}$ & $\begin{array}{l}\text { Schools, communities with } \\
\text { high/low prevalence, low } \\
\text { intensity groups in Africa }\end{array}$ & $\begin{array}{l}\text { Intervention: dipstick test } \\
\text { Sc. haematobium. } \\
\text { Outcomes: sensitivity and } \\
\text { specificity (95\% CI:), diagnostic } \\
\text { odds ratio (DOR) }\end{array}$ & $\begin{array}{c}\text { Sensitivity / specificity (95\% CI) } \\
\text { Detection of egg-positive urine 81\% (79-83)/89\% } \\
\text { (87-92). } \\
\text { In high-prevalence settings } 80 \%(78-83) / 86 \%(82-90) ; \\
\text { lower in treated population } 72 \%(61-78) / 87 \%(81-94) ; \\
\text { in lower intensity population subgroups 65\% } \\
\text { (58-72)/82\% (76-90) }\end{array}$ \\
\hline $\begin{array}{l}\text { Wang, et al., } \\
2012 \text { [46] }\end{array}$ & $\begin{array}{l}\text { AMSTAR: } 7 / 11 \\
\text { GRADE: very low- to } \\
\text { low-quality evidence }\end{array}$ & $\begin{array}{l}\text { Systematic review and } \\
\text { meta-analysis of RCTs, } \\
\text { retro-/pro-observational } \\
\text { studies }\end{array}$ & $\begin{array}{l}\text { Infected patients with } \\
\text { schistosomiasis in control } \\
\text { programmes in China }\end{array}$ & $\begin{array}{c}\text { Intervention: IHA and ELISA. } \\
\text { Outcomes: true positive rates, } \\
\text { sensitivity /specificity ( } 95 \% \text { CI:), } \\
\text { DOR }\end{array}$ & $\begin{array}{c}\text { Sensitivity / specificity (95\% CI) } \\
\text { IHA } 75.6 \%(74-77) / 73 \%(72-74) \text { ELISA } 84.9 \% \\
(83-87) / 50.4 \%(49.2-51.6) \\
\text { The DOR of IHA was } 9.41 \text { ( } 95 \% \text { CI: 5-18), and ELISA } \\
4.78 \text { (95\% CI: 3.21-7.13) }\end{array}$ \\
\hline \multicolumn{6}{|c|}{ Included primary studies of diagnostic tests to detect schistosomiasis } \\
\hline $\begin{array}{l}\text { Espirito-Santo } \\
\text { et al., } 2015 \\
\text { [57] }\end{array}$ & $\begin{array}{l}\text { QUADAS-2-11/14 } \\
\text { GRADE: very low- to } \\
\text { low-quality evidence }\end{array}$ & $\begin{array}{l}\text { Cross-sectional } \\
\text { epidemiological survey in } \\
\text { areas of low prevalence of } \\
\text { Sc. Mansoni }\end{array}$ & $\begin{array}{l}\text { The estimated sample size } \\
\text { required was } 650 \text { individuals; } \\
\text { Barra Mansa City, Rio de Janeiro } \\
\text { State, Brazil }\end{array}$ & $\begin{array}{l}\text { Intervention: diagnostic assays: } \\
\text { ELISA-IgG/ELISA-IgM/IFT- } \\
\text { IgM/qPCR in faeces. } \\
\text { Outcomes: sensitivity/specificity } \\
\text { ( } 95 \% \text { CI: })\end{array}$ & $\begin{array}{c}\text { Sensitivity /specificity (95\% CI) } \\
\text { KK 13.8\% (4-32)/99.8\% (99.0-100); ELISA-IgG 66.7\% } \\
\text { (48-82)/91.5\% (89-94); ELISA-IgM 81.8\% (64-93)/82\% } \\
\text { (79-85); IFT-IgM 78.8\% (61-91)/87.7\% (84.8-90); qPCR } \\
\text { in faeces 51.7\% (32-71)/92.6\% (90-95); qPCR in serum } \\
\text { 12.1\% (3-28)/99.1\% (98-99) }\end{array}$ \\
\hline $\begin{array}{l}\text { Espirito-Santo } \\
\text { et al., 2014a } \\
\text { [60] }\end{array}$ & $\begin{array}{l}\text { QUADAS-2-12/14 } \\
\text { GRADE: very low- to } \\
\text { low-quality evidence }\end{array}$ & Cross-sectional study & $\begin{array}{l}\text { City of Barra Mansa, Rio de } \\
\text { Janeiro State, Brazil, with an } \\
\text { estimated prevalence of } 1 \%\end{array}$ & $\begin{array}{l}\text { Intervention: diagnostic assays: } \\
\text { ELISA-IgG and ELISA-IgM. } \\
\text { Outcomes: sensitivity/specificity } \\
\text { (95\% CI:); PPV, NPV }\end{array}$ & $\begin{array}{c}\text { Sensitivity/specificity (95\%CI) } \\
\text { ELISA-IgG 60.0\% (15-95) /89.1\% (86.2-91.5); } \\
\text { ELISA-IgM 60.0(15-95)/79.2\% (75.6-82.5) } \\
\text { PPV/NPV (95\%CI): } \\
\text { ELISA-IgG 4.6\% (1-13) /99.6\% (98-100); ELISA-IgM } \\
\text { 2.5\% (0.5-7); NPV 99.6\% (98.4-100.0) }\end{array}$ \\
\hline
\end{tabular}


Table 1. Cont

\begin{tabular}{|c|c|c|c|c|c|}
\hline Study & Quality & Design & Population & Intervention/Outcomes & Results \\
\hline $\begin{array}{l}\text { Espirito-Santo } \\
\text { et al., 2014b } \\
\text { [56] }\end{array}$ & $\begin{array}{l}\text { QUADAS-2-13/14 } \\
\text { GRADE: very low- to } \\
\text { low-quality evidence }\end{array}$ & $\begin{array}{l}\text { Cross-sectional } \\
\text { epidemiological survey }\end{array}$ & $\begin{array}{l}7000 \text { inhabitants located in the } \\
\text { outskirts of Barra Mansa, Rio de } \\
\text { Janeiro, Brazil }\end{array}$ & $\begin{array}{l}\text { Intervention: } \mathrm{qPCR} \text { in serum or } \\
\text { faeces. } \\
\text { Outcomes: sensitivity /specificity } \\
\text { (95\% CI:); PPV, NPV }\end{array}$ & $\begin{array}{c}\text { Sensitivity / specificity }(95 \% \mathrm{CI}) \\
\text { qPCR in faeces 80.0\% (28-99)/92.4\% (90-94); } \mathrm{qPCR} \text { in } \\
\text { serum 20.0\% (0.5-71.6)/98.8 (97-99) } \\
\text { PPV/NPV (95\% CI:): } \\
\text { qPCR in faeces 8.0\% (2-19)/99.8\% (99-100); } \mathrm{qPCR} \text { in } \\
\text { serum } 12.5 \%(0.3-52.7) / 99.3 \%(98.2-99.8)\end{array}$ \\
\hline $\begin{array}{l}\text { Lodh et al., } \\
2013 \text { [55] }\end{array}$ & $\begin{array}{l}\text { QUADAS-2-12/14 } \\
\text { GRADE: very low- to } \\
\text { low-quality evidence }\end{array}$ & Cross-sectional case study & $\begin{array}{l}\text { Filtered urine specimens from } \\
\text { infected and not-infected patients } \\
\text { in Zambia }\end{array}$ & $\begin{array}{l}\text { Intervention: qPCR ELISA IgG in } \\
\text { serum or faeces; filtered Urine PCR. } \\
\text { Outcomes: sensitivity /specificity } \\
\text { (95\% CI:); PPV, NPV }\end{array}$ & $\begin{array}{c}\text { Sensitivity / specificity (95\%CI) } \\
\text { KK test 57\% (45-68)/100\% (69-100); CCA rapid test 65\% } \\
(56-77) / 60 \%(26-88) ; \text { PCR 100\% (95-100)/100\% (69-100) } \\
\text { PPV/NPV: } \\
\text { KK test 100\%/23\%; CCA rapid test 93\%/19\%; PCR } \\
\text { 100\%/100\%. }\end{array}$ \\
\hline $\begin{array}{l}\text { Kinkel et al., } \\
2012[54]\end{array}$ & $\begin{array}{l}\text { QUADAS-2-12/14 } \\
\text { GRADE: very low- to } \\
\text { low-quality evidence }\end{array}$ & $\begin{array}{l}\text { Retrospective } \\
\text { comparative diagnostic } \\
\text { study: performance of } 8 \\
\text { serological tests for } \\
\text { Schistosoma spp }\end{array}$ & $\begin{array}{l}\text { Serum specimens from infected } \\
\text { patients and those without the } \\
\text { infection in low-prevalence } \\
\text { locations or non-endemic settings } \\
\text { (Germany) }\end{array}$ & $\begin{array}{l}\text { Intervention: serological assays: } \\
\text { IFAT, ELISA-CA, ELISA-AWA, } \\
\text { ELISA-SEA, IHA, ELISA-NovaTec, } \\
\text { ELISA-DRG and ELISA-Viramed. } \\
\text { Outcomes: sensitivity and } \\
\text { specificity ( } 95 \% \text { CI:) }\end{array}$ & $\begin{array}{c}\text { Sensitivity/specificity-(95\% CI): } \\
\text { IFAT 75.7\% (58-98)/98.1\% (92-99); ELISA-CA 40.5\% } \\
\text { (25-59)/95.2\% (89-98); ELISA-AWA 54.1\% } \\
(37-70) / 100 \% \text { (95.6-100); ELISA-SEA-75.7\% } \\
\text { (58-98)/97.1\% (91-99); IHA 73.0\% } \\
\text { (55.6-85.6)/99.0\% (94.0-100); ELISA-NovaTec } 64.9 \% \\
\text { (47-79)/99 (94-100); ELISA-DRG 78.3\% (61.3-89.6)/88.4 } \\
\text { (80-94); ELISA-Viramed 67.6\% (50-81)/76.9\% (67-84). }\end{array}$ \\
\hline $\begin{array}{l}\text { De Frotas et } \\
\text { al., } 2011[58]\end{array}$ & $\begin{array}{l}\text { QUADAS-2-12/14 } \\
\text { GRADE: very low- to } \\
\text { low-quality evidence }\end{array}$ & Cross-sectional survey & $\begin{array}{l}\text { Stool and serum specimens from } \\
\text { infected and not infected patients, } \\
\text { low-endemic setting in Brazil }\end{array}$ & $\begin{array}{l}\text { Intervention: serological assays, } \\
\text { ELISA IgG } \\
\text { Outcomes: sensitivity and } \\
\text { specificity }(95 \% \mathrm{CI})\end{array}$ & $\begin{array}{c}\text { Sensitivity / specificity (95\% CI): } \\
\text { ELISA-IgG 100\% (68-100)/72.9\% (67-78). } \\
\text { PPV/NPV (95\% CI): } \\
\text { ELISA-IgG 26.0\% (18-36) / } 100 \%(97-100) .\end{array}$ \\
\hline $\begin{array}{l}\text { Silveira et al., } \\
2016[59]\end{array}$ & $\begin{array}{l}\text { QUADAS-2-12/14 } \\
\text { GRADE: very low- to } \\
\text { low-quality evidence }\end{array}$ & $\begin{array}{l}\text { Evaluation of the CCA } \\
\text { test to diagnose } \\
\text { Sc. mansoni in Minas } \\
\text { Gerais State, Brazil. }\end{array}$ & $\begin{array}{l}\text { Infected individuals in regions } \\
\text { with moderate to high prevalence }\end{array}$ & $\begin{array}{c}\text { Intervention: } \\
\text { CCA-immuno-chromatographic } \\
\text { test. } \\
\text { Outcomes: sensitivity } / \text { specificity } \\
\text { (95\% CI: })\end{array}$ & $\begin{array}{c}\text { Sensitivity / specificity (95\% CI): } \\
\text { CCA-ICT 68.7\% (54-81)/97.6\% (87-99) }\end{array}$ \\
\hline $\begin{array}{l}\text { Beltrame et } \\
\text { al., } 2017 \text { [61] }\end{array}$ & $\begin{array}{l}\text { QUADAS-2-12/14 } \\
\text { GRADE: very low- to } \\
\text { low-quality evidence }\end{array}$ & $\begin{array}{l}\text { Accuracy of } \\
\text { parasitological and } \\
\text { immunological tests for } \\
\text { the screening of human } \\
\text { schistosomiasis in } \\
\text { immigrants and refugees } \\
\text { from African countries }\end{array}$ & $\begin{array}{l}\text { Frozen serum specimens from } \\
\text { recent African asylum seekers } \\
\text { that were routinely screened for } \\
\text { schistosomiasis in Italy }\end{array}$ & $\begin{array}{c}\text { Intervention: urine CCA; } \\
\text { Bordier-ELISA, Western Blot IgG, } \\
\text { ICT IgG-IgM, microscopy } \\
\text { compared with composite reference } \\
\text { standard. } \\
\text { Outcomes: sensitivity/specificity } \\
\text { (95\% CI:) }\end{array}$ & $\begin{array}{c}\text { Sensitivity /specificity (95\% CI): } \\
\text { Urine CCA 29\% (22-37)/95\% (91-97); Bordier-ELISA } \\
71 \% \text { (63-78)/99.6\% (98-100); Western blot IgG 92\% } \\
\text { (86-96)/94\% (90-97); ICT IgG-IgM 96\% (91-99)/83\% } \\
\text { (77-87); microscopy 45\% (37-54)/100\% }\end{array}$ \\
\hline \multicolumn{6}{|c|}{ Included systematic reviews for diagnostic effectiveness for strongyloidiasis } \\
\hline $\begin{array}{l}\text { Campo } \\
\text { Polanco et } \\
\text { al., } 2014 \text { [51] }\end{array}$ & $\begin{array}{l}\text { AMSTAR: } 11 / 11 \\
\text { GRADE: } \\
\text { moderate-quality } \\
\text { evidence }\end{array}$ & $\begin{array}{l}\text { Systematic review and } \\
\text { meta-analysis }\end{array}$ & $\begin{array}{l}\text { Individuals with active/chronic } \\
\text { infection }\end{array}$ & $\begin{array}{l}\text { Intervention: Baermann method, } \\
\text { agar plate, direct faecal smear } \\
\text { examination and formol-ether } \\
\text { concentration technique. } \\
\text { Outcome: sensitivity and specificity } \\
\text { ( } 95 \% \text { CI: })\end{array}$ & $\begin{array}{l}\text { Sensitivity: Baermann method }(72 \%) \text { with } L R+228 \text { and } \\
\text { LR-0.32; APC } 89 \%, \text { LR+341 and LR- } 0.11 \text {; stool } \\
\text { microscopy } 21 \% \text {, LR + } 67 \text { and LR }-0.67 \text {; formol-ether } \\
\text { concentration } 48 \% \text {, LR + } 110 \text { and LR-0.59. } \\
\text { Specificity: } 100 \% \text { in all four tests. APC and Baermann } \\
\text { method are best. }\end{array}$ \\
\hline
\end{tabular}


Table 1. Cont

\begin{tabular}{|c|c|c|c|c|c|}
\hline Study & Quality & Design & Population & Intervention/Outcomes & Results \\
\hline \multicolumn{6}{|c|}{ Included systematic reviews of diagnostic tests to detect schistosomiasis } \\
\hline $\begin{array}{l}\text { Requena-Méndez } \\
\text { et al., [19] }\end{array}$ & $\begin{array}{l}\text { AMSTAR: } 7 / 11 \\
\text { GRADE: low- to } \\
\text { moderate-quality } \\
\quad \text { evidence }\end{array}$ & Systematic review & $\begin{array}{l}\text { Individuals with active/chronic } \\
\text { infection }\end{array}$ & $\begin{array}{c}\text { Intervention: Baermann method, } \\
\text { agar plate, direct faecal smear } \\
\text { examination and formol-ether } \\
\text { concentration technique, } \\
\text { serological techniques. } \\
\text { Outcome: sensitivity and specificity } \\
\text { (95\% CI:) }\end{array}$ & $\begin{array}{l}\text { No meta-analysis was undertaken. Sensitivity and } \\
\text { specificity of different techniques were individually } \\
\text { reported. }\end{array}$ \\
\hline \multicolumn{6}{|c|}{ Included primary studies for diagnostic effectiveness for strongyloidiasis } \\
\hline $\begin{array}{l}\text { Bisofi et al., } \\
2014 \text { [62] }\end{array}$ & $\begin{array}{l}\text { QUADAS-2: } 13 / 14 \\
\text { GRADE: low-quality } \\
\text { evidence }\end{array}$ & $\begin{array}{l}\text { Retrospective } \\
\text { comparative diagnostic } \\
\text { study to evaluate the } \\
\text { performance of } 5 \text { tests for } \\
\text { St. stercoralis. }\end{array}$ & $\begin{array}{l}\text { Serum specimens from subjects } \\
\text { with St. stercoralis; healthy people } \\
\text { and patients with previous } \\
\text { exposure }\end{array}$ & $\begin{array}{c}\text { Intervention: IFAT, NIE-LIPS } \\
\text { NIE-ELISA, IVD-ELISA- and } \\
\text { Bordier-ELISA } \\
\text { Outcome: sensitivity and specificity } \\
\text { ( } 95 \% \text { CI:) }\end{array}$ & $\begin{array}{c}\text { Sensitivity / specificity (95\% CI): } \\
\text { NIE-ELISA 75.4\% (67-83)/94.8\% (91-99); NIE-LIPS } \\
\text { 85.1\% (78-92)/100\% (100-100); IFAT 93.9\% } \\
\text { (89-98)/92.2\% (87-97); IVD-ELISA 91.2\% (86-96)/99.1\% } \\
\text { (97.4-100.0); Bordier-ELISA 89.5\% (84-95) 98.3\% } \\
\text { (96-100). }\end{array}$ \\
\hline $\begin{array}{l}\text { Rascoe et al., } \\
2015 \text { [63] }\end{array}$ & $\begin{array}{l}\text { QUADAS-2: } 10 / 14 \\
\text { GRADE: low-quality } \\
\text { evidence }\end{array}$ & $\begin{array}{l}\text { Retrospective } \\
\text { comparative diagnostic } \\
\text { study of } 5 \text { tests for the } \\
\text { follow-up of patients } \\
\text { infected with St. stercoralis }\end{array}$ & $\begin{array}{l}\text { Serum samples positive for } S t \text {. } \\
\text { stercoralis and negative samples } \\
\text { from United States residents with } \\
\text { no history of foreign travel }\end{array}$ & $\begin{array}{l}\text { Intervention: Ss-NIE-1 ELISA, } \\
\text { Ss-NIE-1 Luminex. } \\
\text { Outcome: sensitivity and specificity } \\
\text { (95\% CI:) }\end{array}$ & $\begin{array}{l}\text { Sensitivity / specificity (95\% CI): } \\
\text { SS-NIE-1 ELISA 95\% (92-97)/93\% (90-96); Ss-NIE-1 } \\
\text { Luminex 93\% (88-96)/95\% (93-97). } \\
\text { The inter-assay coefficient of variation was determined } \\
\text { to be 22\% for the low-positive control serum and 10\% } \\
\text { for the medium-positive control serum. }\end{array}$ \\
\hline $\begin{array}{l}\text { Knopp et al., } \\
2014 \text { [64] }\end{array}$ & $\begin{array}{l}\text { QUADAS-2: } 11 / 14 \\
\text { GRADE: low-quality } \\
\text { evidence }\end{array}$ & $\begin{array}{l}\text { International standard } \\
\text { randomised controlled } \\
\text { trial }\end{array}$ & $\begin{array}{l}\text { Children and adults residing in } \\
\text { rural villages in the Baga moyo } \\
\text { District, Tanzania (endemic areas) }\end{array}$ & $\begin{array}{c}\text { Intervention: Real-time PCR, } \\
\text { FLOTAC technique, KK method. } \\
\text { Outcome: sensitivity and specificity } \\
\text { (95\% CI:) }\end{array}$ & $\begin{array}{c}\text { Sensitivity/specificity (95\% CI): } \\
\text { PCR + pseudo-standard PCR 17.4 (8-31)/3.9 (89-97); } \\
\text { Baermann + pseudo-standard } 47 \text { (23-72)/78.4 (72-84); } \\
\text { PCR + multiple gold standard } 30.9(19.1-44.8) / 100 \\
\text { (100-100); Baermann + multiple gold standard } 83.6 \\
\text { (71.2-92.2)/100 (100-100) }\end{array}$ \\
\hline
\end{tabular}

AWA: adult worm antigen; AMSTAR: a tool for assessing the methodological quality of systematic reviews; APC: agar plate culture; CA: Cercarial antigen; CCA: circulatory cathodic antigen; CI: confidence interval; DOR: diagnostic odds ratio; GRADE: Grading of Recommendations, Assessment, Development and Evaluation; ELISA: enzyme-linked immunosorbent assay; FLOTAC: novel multivalent faecal egg count method; ICT: Immuno chromatographic test; IFAT: indirect fluorescent antibody technique; IHA: indirect haemagglutination: In Vitro Diagnostic kit; KK: Kato-Katz method; LIPS: luciferase immunoprecipitation system; LR+: positive likelihood ratio; LR-: negative likelihood ratio; NIE: a 31-kDa recombinant antigen; NovaTec: NovaTec Immundiagnostica, Dietzenbach, Germany; NPV: negative predictive value; POC: point-of-care; qPCR: quantitative PCR (real-time polymerase chain reaction); PPV: positive predictive value; RCT: randomised controlled trial; SEA: soluble egg antigen; Ss-NIE-1: a luciferase tagged recombinant protein of St. stercoralis for IgG and IgG specific antibodies; QUADAS-2: a tool for the quality assessment of diagnostic accuracy studies; Viramed ${ }^{\circledR}$ : Viramed Biotech, Planegg, Germany). 
Table 2. Characteristics of included studies about efficacy of treatment for schistosomiasis and strongyloidiasis, $1993-2016$

\begin{tabular}{|c|c|c|c|c|c|}
\hline Study & Quality & Design & Population & Intervention/Outcomes & Results \\
\hline \multicolumn{6}{|c|}{ Treatment efficacy of anti-Schistosoma drugs } \\
\hline $\begin{array}{l}\text { Kramer et al., } \\
\quad 2014 \text { [48] }\end{array}$ & $\begin{array}{l}\text { AMSTAR: } 11 / 11 \\
\text { Data in study: } \\
\text { GRADE: high-quality } \\
\text { evidence }\end{array}$ & $\begin{array}{l}\text { Systematic review, } \\
\text { fixed effects meta-analysis; } \\
\text { Embase, MEDLINE (1966 } \\
\text { to 2014), LILACS, } \\
\text { Cochrane library, } \\
\text { Cochrane infectious } \\
\text { disease (1980-2014) }\end{array}$ & $\begin{array}{l}\text { School-aged and young adults: } \\
\text { 6-20 years (16 trials); } 2-23 \text { years } \\
\text { (5 trials); Adults ( } 2 \text { trials). } \\
\text { Participants setting: Rural areas } \\
\text { in } 15 \text { sub-Saharan African } \\
\text { countries; an urban setting in } \\
\text { Saudi Arabia }\end{array}$ & $\begin{array}{l}\text { Interventions: drugs used to treat } \\
\text { urinary schistosomiasis: praziquantel, } \\
\text { metrifonate, artesunate and/or in } \\
\text { combination } \\
\text { Outcome: parasitological cure or } \\
\text { failure at } 4 \text { weeks; \% egg reduction rate } \\
\text { at } 4 \text { weeks }\end{array}$ & $\begin{array}{l}\text { Praziquantel (single dose } 40 \mathrm{mg} / \mathrm{kg} \text { ), egg reduction }(60 \%) \\
\text { in urine achieved in } 4-8 \text { weeks ( } 38 \text { per } 100 \text { ( } 95 \% \text { CI: } 26-54) \text { ). } \\
\text { Treatment failure: RR } 0.42,(95 \% \text { CI: } 0.29-0.59) \text {, } 864 \\
\text { participants, } 7 \text { trials } \\
\text { Metrifonate (single dose } 10 \mathrm{mg} / \mathrm{kg} \text { ) reduced egg excretion } \\
\text { only marginally in comparison to placebo (RR 0.63, } 95 \% \text { CI: } \\
0.54 \text { to } 0.73 \text { ) } 210 \text { participants, } 1 \text { trial, at } 8 \text { months }\end{array}$ \\
\hline $\begin{array}{l}\text { Danso-Appiah } \\
\text { et al., 2013 [47] }\end{array}$ & $\begin{array}{l}\text { AMSTAR: } 11 / 11 \\
\text { Data in study: } \\
\text { GRADE: low- to } \\
\text { moderate-quality } \\
\text { evidence }\end{array}$ & $\begin{array}{c}\text { Systematic review and } \\
\text { meta-narrative of RCTs, } \\
\text { RTCs of anti-Schistosoma } \\
\text { drugs }\end{array}$ & $\begin{array}{c}\text { Trials conducted in Africa } \\
(n=36) \text {, South America }(n=15 ; \\
\text { all in Brazil) and the Middle } \\
\text { East }(n=1) . \\
52 \text { trials enrolling } 10,269 \\
\text { participants in endemic areas }\end{array}$ & $\begin{array}{l}\text { Intervention: praziquantel } 40 \mathrm{mg} / \mathrm{kg} \text {, } \\
\text { oxamniquine } 40 \mathrm{mg} / \mathrm{kg}\end{array}$ & $\begin{array}{l}\text { Praziquantel (single dose } 40 \mathrm{mg} / \mathrm{kg} \text { ) vs. placebo: reduced } \\
\text { parasitological treatment failure at } 1 \text { month }(69 / 100 ; \mathrm{RR}= \\
3.13,2 \text { trials, } 414 \mathrm{participants}) \text {. } \\
\text { Praziquantel (single dose } 30 \mathrm{mg} / \mathrm{kg}) \text { : } \mathrm{RR}=1.52,3 \text { trials, } 521 \\
\text { participants. } \\
\text { Higher doses: no significant difference. } \\
\text { Oxamniquine (single dose } 40 \mathrm{mg} / \mathrm{kg} \text { ) vs. Placebo: reduced } \\
\text { parasitological treatment failure at } 3 \text { months in } 2 \text { trials } \\
\text { (68/100; } \mathrm{RR}=8.74) \text {. }\end{array}$ \\
\hline $\begin{array}{l}\text { Pérez del Villar } \\
\text { et al., 2012 [49] }\end{array}$ & $\begin{array}{l}\text { AMSTAR: } 11 / 11 \\
\text { Data in study: } \\
\text { not reported. } \\
\text { GRADE: } \\
\text { Moderate-quality } \\
\text { evidence }\end{array}$ & $\begin{array}{l}\text { Quantitative systematic } \\
\text { review and meta-analysis }\end{array}$ & $\begin{array}{l}\text { Healthy villagers who live in } \\
\text { areas in Africa endemic for } \\
\text { Sc. haematobium and Sc. mansoni } \\
\text { and in China for Sc. Japonicum }\end{array}$ & $\begin{array}{l}\text { Intervention: prophylactic effect of } \\
\text { artesunate or artemether vs. placebo } \\
\text { against } S c \text {. haematobium, Sc. mansoni } \\
\text { and Sc. japonica infections. } \\
\text { Outcomes: parasitological cure rate at } \\
\text { 3-8 weeks; infection rate at } 3-4 \text { weeks } \\
\text { after treatment. }\end{array}$ & $\begin{array}{l}\text { Artesunate treatment (single dose: significantly lower cure } \\
\text { rates than with praziquantel. } \\
\text { Combined therapy of artesunate plus } \\
\text { sulfadoxine-pyrimethamine: significantly less effective than } \\
\text { praziquantel treatment } \\
\text { Combination of artemisinin derivatives and praziquantel: } \\
\text { higher cure rate than praziquantel monotherapy } \\
\text { Artesunate or artemether: significantly better than a } \\
\text { placebo. }\end{array}$ \\
\hline \multicolumn{6}{|c|}{ Treatment efficacy of drugs for strongyloidiasis } \\
\hline $\begin{array}{l}\text { Henriquez- } \\
\text { Camacho et al., } \\
2016 \text { [52] }\end{array}$ & $\begin{array}{l}\text { AMSTAR: 11/11 } \\
\text { GRADE: } \\
\text { Moderate-quality } \\
\text { evidence }\end{array}$ & $\begin{array}{l}\text { Systematic review of } \\
\text { RCTs, controlled or } \\
\text { uncontrolled } \\
\text { interventional studies. }\end{array}$ & $\begin{array}{c}\text { Individuals with chronic } \\
\text { infections of St. stercoralis; } \\
\text { Immuno-competent patients. } \\
\text { All ages }\end{array}$ & $\begin{array}{c}\text { Intervention: ivermectin } \\
\text { (single/double dose) vs. albendazole } \\
\text { or thiabendazole. } \\
\text { Outcome: elimination of infection; } \\
\text { parasitological cure }(>2 \text { negative stool } \\
\text { samples, } 5 \text { weeks). }\end{array}$ & $\begin{array}{c}\text { Ivermectin (single/double dose) vs. albendazole: } \\
\text { parasitological cure was higher with ivermectin, } 84 / 100 \mathrm{vs.} \\
48 / 100 \text { ivermectin }(\mathrm{RR}=1.79) \text {. Ivermectin vs. } \\
\text { thiabendazole: little or no difference in parasitological cure, } \\
74 / 100 \text { vs. } 68 / 100), \text { but adverse events were less common } \\
\text { with ivermectin (RR }=0.31 \text { ) than albendazole. No serious } \\
\text { adverse events or death reported }\end{array}$ \\
\hline
\end{tabular}

Literature in Health Sciences; RCT: randomized clinical trial; RR: Relative Risk. 
Table 3. Characteristics of included studies on cost-effectiveness of screening and treatment of schistosomiasis and strongyloidiasis, 1993-2016.

\begin{tabular}{|c|c|c|c|c|c|}
\hline Study & Quality & Design & Population & Intervention/Outcomes & Results \\
\hline $\begin{array}{l}\text { Libman et al., } \\
1993 \text { [70] }\end{array}$ & NA & $\begin{array}{l}\text { Retrospective-cross-sectional } \\
\text { study with cost analysis }\end{array}$ & $\begin{array}{l}\text { Cohort of individuals returning } \\
\text { from the tropics and screened } \\
\text { in a Canadian clinic 1981-1987 } \\
\text { Costs in 1988 CAD }\end{array}$ & $\begin{array}{l}\text { Stool examination + eosinophil count + } \\
\text { serological studies for filariasis and } \\
\text { schistosomiasis (gold standard); vs. } \\
\text { stool examination + eosinophil count; } \\
\text { vs. stool examination alone; vs. stool } \\
\text { examination + serological studies; vs. } \\
\text { eosinophil counts only } \\
\text { Outcome: difference in cost or resource } \\
\text { use/cost effectiveness }\end{array}$ & $\begin{array}{l}\text { Difference in resource use/costs: high-/low-prevalence } \\
\text { locations } \\
\text { Costs per case of schistosomiasis and/or } \\
\text { strongyloidiasis diagnosed for each strategy: (i) } \\
\text { CAN } \$ 4674 \text { [€3693]; (ii) CAN\$6111 [€4829]; (iii) } \\
\text { CAN } \$ 4788 \text { [€3783]; (iv) CAN\$3737 [€2953]; (v) } \\
\text { CAN\$3307 [€2613] } \\
\text { Cost-effectiveness (ICER or INB): no ICER calculated. } \\
\text { Study did not include a decision analytic model }\end{array}$ \\
\hline $\begin{array}{l}\text { Muennig et al., } \\
1999 \text { [66] }\end{array}$ & NA & Decision analytic model & $\begin{array}{l}\text { Large immigrant populations in } \\
\text { which St. stercoralis is not } \\
\text { endemic (one third of the } \\
\text { sample population was from } \\
\text { the state of New York) } \\
\text { Costs in } 1997 \text { USD }\end{array}$ & $\begin{array}{l}\text { No preventive intervention (watchful } \\
\text { waiting) vs. universal screening vs. } \\
\text { presumptive treatment with } \\
\text { albendazole } \\
\text { Outcome: difference in cost or resource } \\
\text { use/cost effectiveness (ICER or INB) } \\
\text { per DALY averted }\end{array}$ & $\begin{array}{l}\text { Difference in resource use/costs: gross costs: USD } \\
11,086,181[€ 7,228,785] \text { for no intervention, USD } \\
7,290,624 \text { [€40,203,726] per year for treatment with } \\
\text { albendazole, USD 40,547,651 [€40,203,726] for universal } \\
\text { screening } \\
\text { Cost-effectiveness (ICER or INB): treatment with } \\
\text { albendazole was cost saving compared with no } \\
\text { intervention, universal screening had ICER of USD } \\
\text { 159,236/DALY [€157,885/DALY] averted }\end{array}$ \\
\hline $\begin{array}{l}\text { Muennig et al., } \\
2004 \text { [67] }\end{array}$ & NA & $\begin{array}{l}\text { Decision analytic model } \\
\text { (Markov) }\end{array}$ & $\begin{array}{l}\text { California and New York, two } \\
\text { states with large immigrant } \\
\text { populations in which St. } \\
\text { stercoralis is not endemic } \\
\text { Costs in } 2000 \text { USD }\end{array}$ & $\begin{array}{l}\text { Intervention: no intervention (watchful } \\
\text { waiting) vs. } 3 \text { or } 5 \text { days of albendazole } \\
\text { vs. eosinophil screening vs. ivermectin } \\
\text { Outcome: difference in cost or resource } \\
\text { use/ cost effectiveness (ICER or INB) }\end{array}$ & $\begin{array}{c}\text { Difference in resource use/costs: costs per person: no } \\
\text { intervention USD 1666 [€1611], albendazole } 3 \text { days USD } \\
1674 \text { [€1618], albendazole 5 days USD } 1680 \text { [€1624], } \\
\text { screening USD 1684 [€1628], ivermectin USD } 1688 \\
\text { [€1632] } \\
\text { Cost-effectiveness (ICER or INB): ICERs varied based } \\
\text { on prevalence: albendazole USD 155-1584/QALY } \\
\text { gained [€150-1531], albendazole } 5 \text { days USD } \\
\text { 314-3175/QALY gained [€304-3069], ivermectin USD } \\
\text { 848-8514/QALY gained [€820-8231]. Eosinophil was } \\
\text { documented among all prevalence groups }\end{array}$ \\
\hline $\begin{array}{l}\text { King et al., } \\
2011 \text { [65] }\end{array}$ & AMSTAR & $\begin{array}{c}\text { Systematic review of efficacy of } \\
\text { schistosomiasis treatment with } \\
\text { praziquantel (by dose), with a } \\
\text { Markov model estimating } \\
\text { cost-effectiveness of various } \\
\text { dosing strategies }\end{array}$ & $\begin{array}{l}\text { Non-migrants in endemic } \\
\text { setting; population-based or } \\
\text { sub-population-based (e.g.,. } \\
\text { schools) drug treatment of } \\
\text { Sc. haematobium or Sc. Mansoni. } \\
\text { Costs in } 2002 \text { \& } 2008 \text { USD }\end{array}$ & $\begin{array}{l}\text { Intervention: No treatment vs. single } \\
\text { dose of praziquantel per annual } \\
\text { treatment vs. double dose } \\
\text { Outcome: difference in cost or resource } \\
\text { use/cost effectiveness (ICER or INB) }\end{array}$ & $\begin{array}{l}\text { Difference in resource use/costs: single dose lifetime } \\
\text { cost: USD } 23 \text { [€19] per person; double dose: USD } 46 \\
\text { [€35] per person. } \\
\text { Cost-effectiveness (ICER or INB): single dose: ICER of } \\
\text { USD } 48 \text { [€39] and USD 46 [€37] per QALY gained for Sc } \\
\text { mansoni and Sc. haematobium, respectively, compared } \\
\text { with no treatment; double dose: ICERs of USD } 291 \\
\text { [€236] and USD } 433 \text { [€351] per QALY gained } \\
\text { respectively compared with single dose }\end{array}$ \\
\hline
\end{tabular}


Table 3. Cont

\begin{tabular}{|c|c|c|c|c|c|}
\hline Study & Quality & Design & Population & Intervention/Outcomes & Results \\
\hline $\begin{array}{l}\text { Worrell et al., } \\
2015 \text { [69] }\end{array}$ & NA & Cost analysis study & $\begin{array}{l}\text { Cohort of children in Kenya } \\
\text { assessed 2010-2011. } \\
\text { Non-migrant settings. } \\
\text { Costs in } 2010 \text { USD }\end{array}$ & $\begin{array}{c}\text { Intervention: single KK (stool } \\
\text { examination) vs. triplicate KK vs. POC } \\
\text { CCA (urine dipstick) } \\
\text { Outcome: difference in cost or resource } \\
\text { use/cost effectiveness (ICER or INB) } \\
\end{array}$ & $\begin{array}{l}\text { Difference in resource use/ costs: total costs per test: } \\
\text { single KK USD 6.89 [€5], triplicate KK USD 17.54 [€14], } \\
\text { POC CCA USD 7.26 [€6] } \\
\text { Cost-effectiveness (ICER or INB): no ICER calculated, } \\
\text { this was not a decision analysis study. }\end{array}$ \\
\hline $\begin{array}{l}\text { Maskery et al., } \\
2016 \text { [68] }\end{array}$ & NA & $\begin{array}{l}\text { Cost analysis study; Markov } \\
\text { model: discount rate of } 3 \% \text { over } \\
60 \text {-year time horizon; costs in } \\
2013 \text { USD }\end{array}$ & $\begin{array}{c}\text { Average annual cohort of } \\
\text { 27,700 Asian refugees based on } \\
\text { Department of } \\
\text { Homeland Security data for } \\
\text { 2002-2011, primarily from } \\
\text { south-east Asia and the Middle } \\
\text { East }\end{array}$ & $\begin{array}{l}\text { Intervention: no screening or treatment } \\
\text { vs. overseas albendazole and } \\
\text { ivermectin treatment vs. domestic } \\
\text { screening and treatment vs. overseas } \\
\text { albendazole and domestic screening } \\
\text { for strongyloidiasis. Outcome: } \\
\text { difference in cost or resource use/cost } \\
\text { effectiveness (ICER or INB) }\end{array}$ & $\begin{array}{c}\text { Difference in resource use/costs, total costs per migrant } \\
\text { (strongyloidiasis.): no treatment USD 5.99 [€5], overseas } \\
\text { albendazole and ivermectin USD 15.12 [€12], domestic } \\
\text { screening and treatment USD 138.36 [€108], overseas } \\
\text { albendazole and domestic screening for Strongyloides } \\
\text { infection USD 78.79 [€61]. } \\
\text { Cost-effectiveness: ICERs per QALY gained: USD } 2219 \\
\text { for "overseas albendazole and ivermectin", USD } 32,706 \\
\text { [€25,422] for domestic screening and treatment, USD } \\
\text { 18,167 [€14,121] for overseas albendazole followed by } \\
\text { domestic screening for strongyloidiasis. All vs. no } \\
\text { screening or treatment [€1723] }\end{array}$ \\
\hline
\end{tabular}

AMSTAR: A measurement tool to assess systematic reviews; CAD: Canadian dollars; CCA: circulatory cathodic antigen; GRADE: Grading of Recommendations, Assessment, Development and Evaluation; ICER: incremental cost-effectiveness ratio, INB: incremental net benefit; NA: Not Applicable KK: Kato-Katz; POC: point-of-care; USD: United States dollars. 


\subsection{Screening: Diagnostic Test Accuracy for Schistosomiasis}

We assessed diagnostic and screening tools for Schistosoma spp. in five included systematic reviews [44-46,50,53] and eight individual studies [54-61]. The best performing tests were included in the GRADE summary of finding on diagnostic tools for screening schistosomiasis (Table 4 and Figure 5).

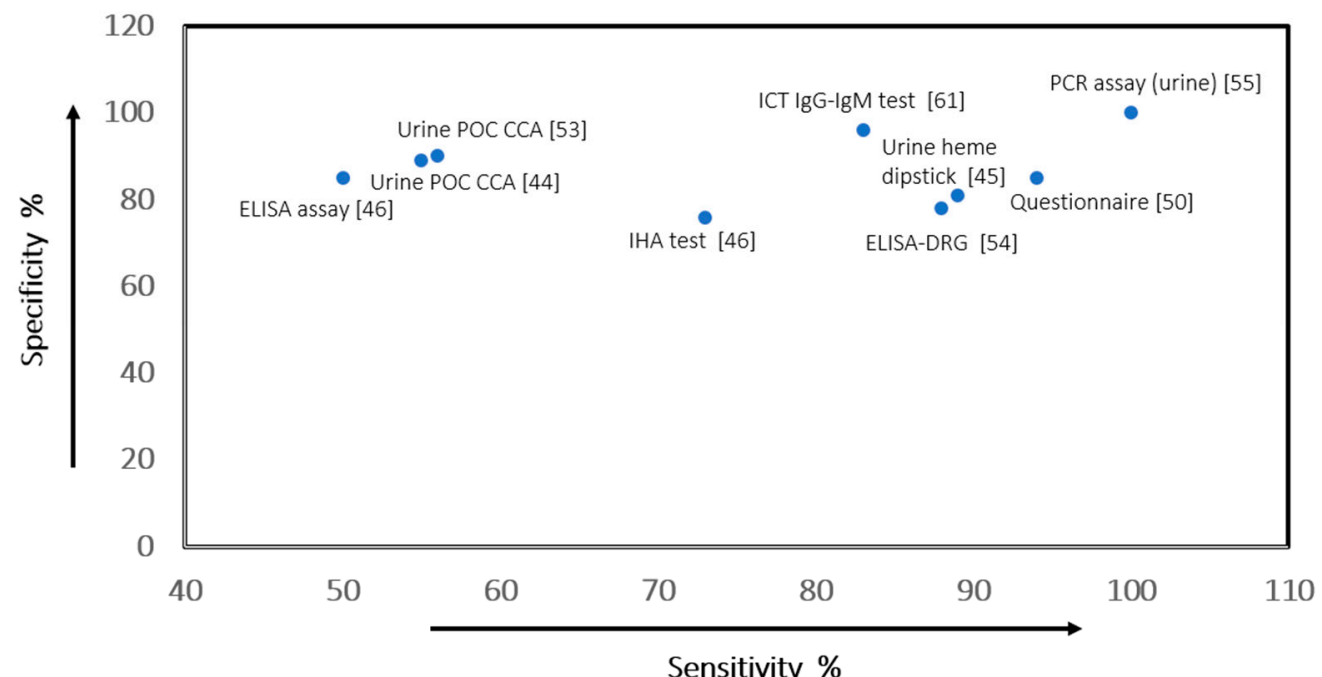

Figure 5. Scatter plot of sensitivity versus specificity values of the Index diagnostic tools for screening schistosomiasis.

\subsubsection{Schistosoma Mansoni}

A meta-analysis reported estimated sensitivity and specificity values of $89 \%$ (95\% CI: 86-92) and 55\% (95\% CI: 46-55) respectively, for the urinary circulating cathodic antigen (CCA) assay that detects Sc. mansoni in endemic areas [44]. Another urinary CCA test for Sc. mansoni [53] reported sensitivity and specificity values of 90\% (95\% CI: 84-94) and 56\% (95\% CI: 39-71), respectively compared with the duplicate Kato-Katz (KK) test (moderate-quality evidence) (Table 4). From the included primary studies, PCR assay in urine was the best-performing diagnostic test for Sc. mansoni with a sensitivity of 100\% (95\% CI: 95-100) compared with the CCA test-65\% (95\% CI: 56-77) and KK test-57\% (95\% CI: 46-68) [55] (very low-quality evidence); the specificity of PCR assay in urine was 100\% (95\% CI: 69-100) (Table 4) [55]. Espírito-Santo et al. reported sensitivity and specificity of 80\% (95\% CI: 28-99) and 92.4\% (95\% CI: 90-94), respectively for quantitative PCR (qPCR) in faeces compared with the KK test (not included in the GRADE Summary of findings) [56].

In low-endemic settings, the best-performing diagnostic test was the IgM-ELISA assay with sensitivity and specificity values of, respectively, 82\% (95\% CI: 64-93) and 82\% (95\% CI: 79-85)-low-quality evidence (Table 1) [57]. In another study, the ELISA-DRG kit showed the best accuracy with sensitivity and specificity values of, respectively, 78\% (95\% CI: 61-90) and 95\% (95\% CI: 89-98) (Table 4) [54]. In a recent study on the accuracy of different screening tests for schistosomiasis in African migrants, the immuno chromatographic test (ICT) IgG-IgM showed the best accuracy, with sensitivity and specificity values of 96\% (95\% CI: 91-99) and 83\% (95\% CI: 77-87) (Table 4) [61]. In all the individual studies, the certainty of evidence was very low to low.

\subsubsection{Schistosoma Haematobium}

The urine heme dipsticks for the diagnosis of Sc. haematobium showed a mean sensitivity and specificity of $81 \%$ (95\% CI: 73-83) and 89\% (95\% CI: 87-92), respectively, and were more accurate in high-prevalence than in low-prevalence settings -low-quality evidence (Table 4) [45]. Similarly, Ochodo et al. reported sensitivity and specificity values of 75\% (95\% CI: 71-79) and 87\% (95\% CI: 
84-90)-low-quality evidence (Table 1) [44]. Furthermore, a meta-analysis on the diagnostic efficiency of questionnaire screening for schistosomiasis reported sensitivity and specificity values of $85 \%$ (95\% CI: 84-86) and 94\% (95\% CI: 94-94) for Sc. haematobium infections (low-quality evidence) (Table 4) [50].

Kinkel et al. evaluated the accuracy of antibody-detection tests for diagnosis of imported Sc. haematobium [54]. The indirect haemagglutination (IHA) test with a sensitivity of $73 \%(95 \%$ CI: 56-86) and specificity of 99\% (95\% CI: 94-100) and the ELISA-DRG with a sensitivity of $78 \%$ (95\% CI: 61-90) and specificity of 95\% (95\% CI: 89-98) demonstrated the best accuracy (certainty of evidence low) (Table 4) [54]. In another study, the ICT IgG-IgM test showed the best accuracy with sensitivity of 96\% (95\% CI: 91-99) and specificity of 83\% (95\% CI: 77-87) (Table 4) [61].

\subsubsection{Schistosoma Japonicum}

In a meta-analysis of the accuracy of antibody detection of Sc. japonicum infection in humans, pooled sensitivities and specificities were 76\% (95\% CI: 74-77) and 73\% (95\% CI: 72-74) for the IHA test and 85\% (95\% CI: 83-87) and 50\% (95\% CI: 49-52) for ELISA (Table 4) [46].

The evidence also suggests that accuracy of diagnostic tests for schistosomiasis depends on pre-test prevalence (Table 5). As prevalence increased (from $2.5 \%$ to $30 \%$ ), the estimated number of false-positives per 1000 migrants tested decreased with all tests-from 47 to 34 (Sc. haematobium/Sc. mansoni) [54], 58 to 42 (Sc. haematobium) [44], 107 to 77 (Sc. Haematobium) [45] and 166 to 119-(Sc. haematobium/Sc. mansoni) [61] per 1000 for ELISA-DRG, questionnaire screening, urine heme dipsticks and ICT IgG-IgM, respectively. The estimated false-negative tests were between 0-6 and $0-73$ per 1000 at $2.5 \%$ and $30 \%$ prevalence for all the tests. At $2.5 \%$ pre-test prevalence, the proportion of correctly diagnosed schistosomiasis infections in migrant populations was $100 \%$ for the urine PCR assay, $96 \%$ for the ICT IgG-IgM test, $90 \%$ for the urine POC CCA, $85 \%$ for the questionnaire screening and $84.9 \%$ for Sc. japonicum ELISA (Table 5).

\subsection{Screening: Diagnostic Test Accuracy for Strongyloidiasis}

We assessed diagnostic and screening tools for St. stercoralis in two included systematic reviews $[19,51]$ and three individual studies (Tables 1 and 6) [62-64]. 
Table 4. GRADE summary of findings on diagnostic tools for screening schistosomiasis, 1993-2017.

\begin{tabular}{|c|c|c|c|c|c|c|c|}
\hline $\begin{array}{l}\text { Index Test at Median Test } \\
\text { Prevalence in Study * }\end{array}$ & $\begin{array}{l}\text { Sensitivity } \\
(95 \% \mathrm{CI})\end{array}$ & $\begin{array}{l}\text { Specificity } \\
(95 \% \mathrm{CI})\end{array}$ & $\begin{array}{c}\text { Post-Test } \\
\text { Probability of a } \\
\text { Positive Result } \\
(95 \% \text { CI })\end{array}$ & $\begin{array}{c}\text { Post-Test } \\
\text { Probability of a } \\
\text { Negative Result } \\
(95 \% \text { CI })\end{array}$ & $\begin{array}{l}\text { Number of } \\
\text { Studies/ } \\
\text { Participants }\end{array}$ & $\begin{array}{l}\text { Certainty of } \\
\text { Evidence } \\
\text { (GRADE) }\end{array}$ & Reference Standard \\
\hline $\begin{array}{l}\text { PCR assay (filtered urine) at } 89 \% \\
\text { prevalence-Sc. mansoni }[55]\end{array}$ & $1.00(0.95-1.00)$ & $1.00(0.69-1.00)$ & $100 \%(96-100)$ & $0 \%(37-0)$ & $1 / 89$ & Very Low $a, b, c$ & KK test-duplicate smears \\
\hline $\begin{array}{l}\text { Urine POC CCA test at } 36 \% \\
\text { prevalence-Sc. mansoni }[44]\end{array}$ & $0.89(0.86-0.92)$ & $0.55(0.46-0.65)$ & $53 \%(47-60)$ & $10 \%(15-7)$ & $15 / 6091$ & Very Low $a, b, c$ & Stool microscopy \\
\hline $\begin{array}{l}\text { Urine POC CCA test at 30\% } \\
\text { prevalence-Sc. mansoni }[53]\end{array}$ & $0.90(0.84-0.94)^{d}$ & $0.56(0.39-0.71)^{d}$ & $47 \%(37-58)$ & $7 \%(15-3)$ & $7 / 4584$ & Moderate $^{a, b}$ & KK test \\
\hline $\begin{array}{l}\text { Questionnaire screening 30\% } \\
\text { prevalence-Sc. haematobium [50] }\end{array}$ & $0.85(0.84-0.86)^{d}$ & $0.94(0.94-0.94)^{d}$ & $86 \%(86-86)$ & $6 \%(7-6)$ & $12 / 41,412$ & Low ${ }^{c, e}$ & Urine filtration/microscopy \\
\hline $\begin{array}{l}\text { ELISA-DRG (commercial kit) at } \\
26 \% \text { prevalence-All cases [54] }\end{array}$ & $0.78(0.61-0.90)$ & $0.88(0.80-0.94)$ & $85 \%(65-95)$ & $7 \%(13-4)$ & $1 / 37$ & Very Low ${ }^{c, e, f}$ & Stool/urine microscopy \\
\hline $\begin{array}{l}\text { Urine heme dipstick at } 27 \% \\
\text { prevalence-Sc. haematobium [45] }\end{array}$ & $0.81(0.73-0.83)^{d}$ & $0.89(0.87-0.92)^{d}$ & $73 \%(67-79)$ & $7 \%(10-6)$ & $98 / 126,119$ & Low ${ }^{a, f, g}$ & Urine microscopy \\
\hline $\begin{array}{c}\text { ELISA at } 24 \% \text { prevalence-Sc. } \\
\text { japonicum [46] }\end{array}$ & $0.85(0.83-0.87)$ & $0.50(0.49-0.52)$ & $35 \%(34-36)$ & $9 \%(10-7)$ & $10 / 9014$ & Low ${ }^{a, f, g}$ & KK and Miracidium hatching test \\
\hline $\begin{array}{l}\text { IHA at } 12 \% \text { prevalence-Sc. } \\
\text { japonicum [46] }\end{array}$ & $0.76(0.72-0.74)^{d}$ & $0.73(0.72-0.74)^{d}$ & $28 \%(26-28)$ & $4 \%(5-5)$ & $15 / 23,411$ & Low $^{a, b}$ & KK and Miracidium hatching test \\
\hline $\begin{array}{l}\text { ICT IgG-IgM test at } 17 \% \\
\text { prevalence Sc. mansoni and } \\
\text { Sc. haematobium [61] }\end{array}$ & $0.96(0.91-0.99)$ & $0.83(0.77-0.87)$ & $13 \%(9-16)$ & $0 \%(0-0)$ & $1 / 373$ & Low $b, c$ & $\begin{array}{l}\text { Stool/urine microscopy/ } \\
\text { composite standard. }\end{array}$ \\
\hline
\end{tabular}

Population: patients with schistosomiasis or stored sera; Settings: high-/low-endemic settings; Target condition: Schistosoma spp. Infections. GRADE: Grading of Recommendations, Assessment, Development and Evaluation. Tests-CCA: circulating cathodic antigen; CI: confidence interval; DRG: DRG Instruments, Marburg, Germany; ELISA: enzyme-linked immunosorbent assay; IHA: indirect haemagglutination; KK: Kato-Katz; POC: point-of-care. ${ }^{*}$ Post-test probability of test was calculated at median test prevalence obtained from individual studies.

a Heterogeneity across similar studies because of several factors; downgraded because of serious inconsistency.

b Use of intermediate or surrogate outcomes rather than health outcomes, hence a source of serious indirectness.

Single study design, not a randomised control trial.

Sensitivity and specificity values obtained from multiple-field study

Use of indirect comparisons; sample population not migrants, another source of indirectness.

Very low-quality of evidence (downgraded by 1 ) because of serious indirectness.

Studies were insufficient to provide summary estimates for CAA tests. 
Table 5. Accuracy of diagnostic tools for schistosomiasis at different pre-test prevalence levels, January 2010-February 2017.

\begin{tabular}{|c|c|c|c|c|c|c|c|c|c|c|c|c|c|}
\hline \multirow{2}{*}{$\begin{array}{c}\text { Index Test } \\
\text { Test } \% \text { Prevalence }^{\text {a }}\end{array}$} & \multicolumn{3}{|c|}{$\begin{array}{c}\text { True Positives } \\
\text { Pre-Test Probability * }\end{array}$} & \multicolumn{3}{|c|}{$\begin{array}{c}\text { False Positives } \\
\text { Pre-Test Probability * }\end{array}$} & \multicolumn{3}{|c|}{$\begin{array}{c}\text { True Negative } \\
\text { Pre-Test Probability * }\end{array}$} & \multicolumn{3}{|c|}{$\begin{array}{c}\text { False Negative } \\
\text { Pre-Test Probability }\end{array}$} & \multirow{2}{*}{$\begin{array}{c}\text { \% Infected } \\
\text { Correctly } \\
\text { Diagnosed }\end{array}$} \\
\hline & $2.5 \%$ & $10 \%$ & $30 \%$ & $2.5 \%$ & $10 \%$ & $30 \%$ & $2.5 \%$ & $10 \%$ & $30 \%$ & $2.5 \%$ & $10 \%$ & $30 \%$ & \\
\hline PCR assay (filtered urine)—Sc. mansoni [55] & 25 & 100 & 300 & 0 & 0 & 0 & 975 & 900 & 700 & 0 & 0 & 0 & $100 \%$ \\
\hline ICT IgG-IgM test-Sc. haematobium/Sc. mansoni [61] & 24 & 96 & 288 & 166 & 153 & 119 & 80 & 747 & & 1 & 4 & 12 & \\
\hline Urine POC CCA test-Sc. mansoni [53] & 23 & 90 & 270 & 429 & 396 & 308 & & 504 & & 2 & 10 & 30 & \\
\hline Questionnaire screening & 21 & 85 & 255 & 58 & 54 & 42 & 917 & 846 & 658 & 4 & 15 & 45 & $85 \%$ \\
\hline $\begin{array}{l}\text { ELISA-DRG (commercial kit)-Sc. } \\
\text { haematobuim } / \text { Sc. mansoni [54] }\end{array}$ & 20 & 78 & 235 & 47 & 43 & 34 & 928 & 857 & 666 & 5 & 22 & 65 & $78.3 \%$ \\
\hline Urine heme dipstick-Sc. haematobium infections [45] & 20 & 81 & 243 & 107 & 99 & 77 & 868 & 801 & 623 & 5 & 19 & 57 & $81.0 \%$ \\
\hline ELISA-Sc. japonicum [46] & 21 & 85 & 255 & 484 & 446 & 347 & 491 & 454 & 353 & 4 & 15 & 45 & $84.9 \%$ \\
\hline IHA-Sc. japonicum [46] & 19 & 76 & 227 & 263 & 243 & 189 & 712 & 657 & 511 & 6 & 24 & 73 & $75.6 \%$ \\
\hline
\end{tabular}

a Different pre-test prevalence or probability of having schistosomiasis in an at-risk population. * Data reported as effect per 1000 migrants tested. Tests: DRG: DRG Instruments, Marburg, Germany; ELISA: enzyme-linked immunosorbent assay; ICT: Immuno chromatographic test; IHA: Indirect haemagglutination; PCR: Polymerase chain reaction assay; POC: Point of care.

Table 6. GRADE summary of findings on diagnostic tools for screening strongyloidiasis, January 1993-February 2017.

\begin{tabular}{|c|c|c|c|c|c|c|c|}
\hline Index Test-at $10 \%$ Prevalence * & $\begin{array}{c}\text { Sensitivity } \\
(95 \% \mathrm{CI})\end{array}$ & $\begin{array}{c}\text { Specificity } \\
\text { (95\% CI) }\end{array}$ & $\begin{array}{c}\text { Post-Test } \\
\text { Probability of a } \\
\text { Positive Result } \\
\text { (95\% CI) } \\
\end{array}$ & $\begin{array}{c}\text { Post-Test } \\
\text { Probability of a } \\
\text { Negative Result } \\
(95 \% \text { CI }) \\
\end{array}$ & $\begin{array}{l}\text { Number of } \\
\text { Studies/ } \\
\text { Participants }\end{array}$ & $\begin{array}{l}\text { Certainty of } \\
\text { Evidence } \\
\text { (GRADE) }\end{array}$ & Reference Standard \\
\hline Baermann method [51] & $0.72(0.67-0.76)^{\mathrm{a}}$ & $1.00(1.00-1.00)^{\mathrm{a}}$ & $100 \%(100-100)$ & $3 \%(4-3)$ & $9 / 2459$ & Moderate $^{b, c}$ & Combination of diagnostic tests \\
\hline Agar plate $-10 \%$ prevalence [51] & $0.89(0.86-0.92)^{\mathrm{a}}$ & $1.00(1.00-1.00)^{\mathrm{a}}$ & $100 \%(100-100)$ & $1 \%(2-1)$ & $10 / 3563$ & Moderate $^{b, c}$ & Combination of diagnostic tests \\
\hline NIE LIPS [62] d & $0.85(0.79-0.92)$ & $0.95(0.93-0.98)$ & $65 \%(56-84)$ & $2 \%(2-1)$ & $1 / 399$ & Low e,f,g & Stool microscopy or culture \\
\hline IVD ELISA—commercial test [62] & $0.92(0.87-0.97)$ & $0.97(0.96-0.99)$ & $77 \%(71-92)$ & $1 \%(1-0)$ & $1 / 399$ & Low e,f,h & Stool microscopy \\
\hline IFAT [62] & $0.94(0.90-0.98)$ & $0.87(0.83-0.91)$ & $45 \%(37-55)$ & $1 \%(1-0)$ & $1 / 399$ & Low ef, h & Stool microscopy and culture \\
\hline Bordier-ELISA — commercial kit [62] & $0.91(0.86-0.96)$ & $0.94(0.91-0.96)$ & $63 \%(52-77)$ & $1 \%(2-0)$ & $1 / 193$ & Low $\mathrm{e}, \mathrm{f}, \mathrm{h}$ & Kato-Katz, Flotac, and Baermann method \\
\hline SS-NIE-1 ELISA [63] & $0.95(0.92-0.97)$ & $0.93(0.90-0.96)$ & $60 \%(71-73 \%)$ & $1 \%(1-0)$ & $1 / 583$ & Low $\mathrm{fg}, \mathrm{i}$ & Stool microscopy and culture \\
\hline
\end{tabular}

Notes: Population: patients with strongyloidiasis or sera infected with St. stercoralis; Settings: low-/high-endemic areas; Target condition: strongyloidiasis (test prevalence 10\%). Cost effectiveness: serological testing may be cost-effective relative to stool and eosinophil testing for both strongyloidiasis and schistosomiasis, because of superior test performance characteristics. Tests: ELISA: enzyme-linked immunosorbent assay; GRADE: Grading of Recommendations, Assessment, Development and Evaluation; IFAT: indirect fluorescent antibody technique; IVD: Invitro diagnostic test; LIPS: luciferase immunoprecipitation system; NIE: a 31-kDa recombinant antigen from St. stercoralis. * Post-test probability of test was calculated at $10 \%$ prevalence for all the tests.

a Sensitivity and specificity values obtained from a multiple-field study.

Evidence was downgraded because of serious inconsistencies and heterogeneity.

Heterogeneity between studies; use of intermediate or surrogate outcomes rather than health outcomes.

Test result with a primary standard.

Absence of a reliable gold standard for diagnosis of S. stercoralis infection. The review did not describe the specific gold standard used in the included studies for each test. Single study design.

Samples were classified according to a composite reference standard, a procedure suggested for evaluation of diagnostic tests when there is no gold standard.

Use of intermediate or surrogate outcomes rather than health outcomes.

The inter-assay coefficient of variation was determined to be $22 \%$ for the low-positive control serum and $10 \%$ for the medium-positive control serum. 
The best conventional diagnostic tools for St. stercoralis have been agar plate culture with a sensitivity and specificity of $89 \%$ (95\% CI: 86-92) and 100\% (95\% CI: 100-100) respectively, and the Baermann method with a sensitivity and specificity of $72 \%$ (95\% CI: 67-76) and 100\% (95\% CI: 100-100) respectively (moderate certainty of evidence) [51]. Knopps et al. reported a much lower sensitivity value of $31 \%$ (95\% CI: 19.1-44.8) for PCR in stools compared with a combination of stool-based methods as the gold standard; specificity was $100 \%$ (95\% CI: 100-100) [64].

Serological antibody detection methods have demonstrated greater sensitivity compared with classical parasitological techniques [19]. Bisoffi et al. reported the accuracy of five serological tests for detection of strongyloidiasis [62]. The sensitivity and specificity values were: $85 \%$ (95\% CI: 79-92) and 100\% (95\% CI: 100-100) for the luciferase-immunoprecipitation system (LIPS) using 31-kD recombinants antigen from St. stercoralis (NIE); 75\% (95\% CI: 66-83) and 95\% (95\% CI: 91-99) for the NIE-ELISA (using the same antigen); $91 \%$ (95\% CI: 86-96) and 99\% (95\% CI: 97-100) for the IVD-ELISA; 90\% (95\% CI: 84-95) and 98\% (95\% CI: 96-100) for the Bordier-ELISA; and 94\% (95\% CI: 90-98) and 92\% (95\% CI: 87-97) for the indirect fluorescent antibody technique (IFAT) (low certainty of evidence) [62] (Figure 6). Rascoe et al. reported comparable values for two new recombinant antigens in antibody detection assays: SS-NIE-1 ELISA with sensitivity of 95\% (95\% CI: 92-97) and specificity of 93\% (95\% CI: 90-96), and Ss-NIE-1 Luminex with sensitivity of 93\% (95\% CI: 86-96) and specificity of 95\% (95\% CI: 93-97) (Table 6) [63].

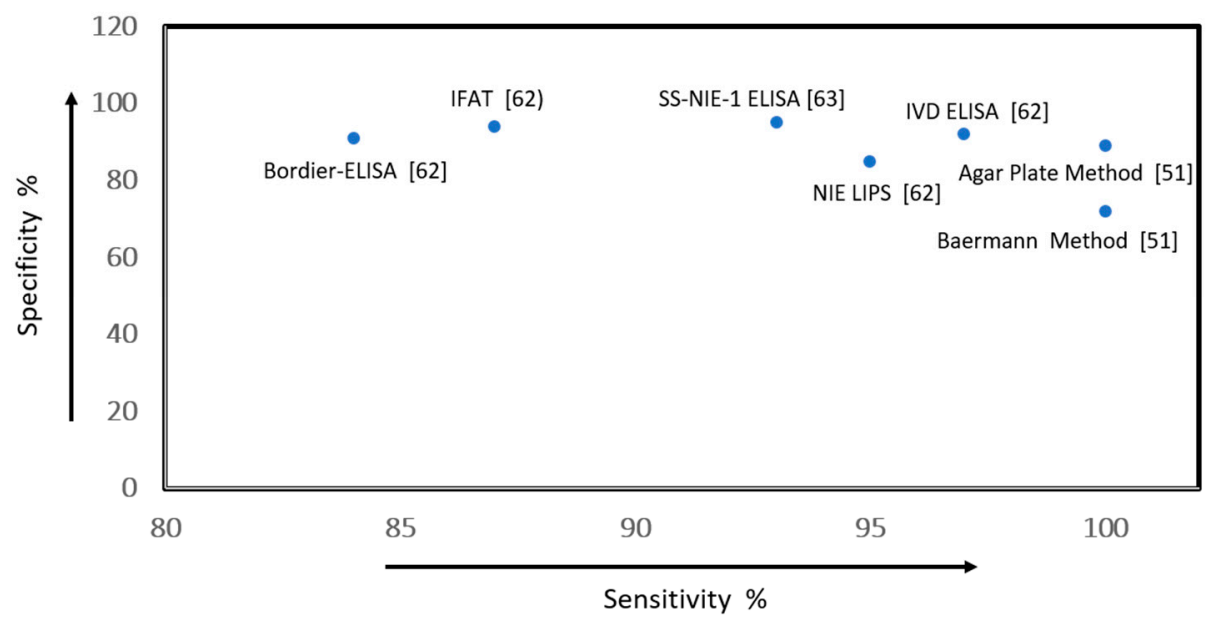

Figure 6. Scatter plot of sensitivity versus specificity values of the Index diagnostic tools for screening strongyloidiasis.

As with schistosomiasis, estimates of false-positive tests per 1000 tested decreased with increasing pre-test prevalence, from 29 to 21, 58 to 42 and 68 to 49 for IVD-ELISA, Bordier-ELISA and SS-NIE-1 ELISA assays, respectively [62,63]. The estimated number of false-positive tests for the Baermann and Agar plate methods was 0 at all pre-test prevalence levels. Lower numbers of false-negatives were estimated for all the serological tests, for example, 1 and 15, and 2 and 24, per 1000 tests for SS-NIE-1 and IVD-ELISA at $2.5 \%$ and $30 \%$ prevalence levels compared with 3 and 33, and 7 and 84 , per 1000 for the Agar plate and Baermann methods. At $2.5 \%$ pre-test prevalence, the proportion of correctly diagnosed Strongyloides infections in migrant populations was $95 \%$ for the SS-NIE-1 ELISA, $93.8 \%$ for IFAT, $92 \%$ for IVD-ELISA and $90.7 \%$ for Bordier-ELISA, compared with $72 \%$ and $89 \%$ for the Baermann and Agar plate methods (Table 7).

\subsection{Treatment Efficacy: Schistosomiasis and Strongyloidiasis}

We evaluated four included systematic reviews on treatment of schistosomiasis and strongyloidiasis (Tables 8 and 9) [47-49,52]. In a Cochrane review, the efficacy of praziquantel (single $40 \mathrm{mg} / \mathrm{kg}$ dose) showed much lower parasitological failure in urine $(<53 \%)$ at 1 to 2 months $(\mathrm{RR}=0.42$; 
95\% CI: 0.29-0.58) compared with placebo [48]. The proportion of people cured with praziquantel varied substantially between trials, from $22.5 \%$ to $83.3 \%$, but was higher than $60 \%$ in five of the seven trials [48]. Similarly, in another Cochrane review, parasitological cure rate for Sc. mansoni infection at one month with praziquantel (single $40 \mathrm{mg} / \mathrm{kg}$ dose) varied substantially across studies, ranging from $52 \%$ to $92 \%$ in Brazil in 2006 and 2007, for example parasitological cure $66 \%$ more in intervention group compared with placebo (RR 3.13; 95\% CI: 1.03-9.53) (Table 8) [47]. Pérez del Villar et al. compared the efficacy of praziquantel and artemisinin derivatives and reported that artesunate showed significantly lower cure rates than praziquantel $30 \%$ vs. $61 \%$ (RR $0.49(0.28-0.75)$ ) [49]. Artemeter monotherapy (6mg $/ \mathrm{kg}$ single dose) reduced Sc. Japonicum infection rates in patients ( $R R=0.25 ; 95 \%$ CI: $0.16-0.40)$. However, a combination of artemisinin derivatives plus praziquantel showed higher cure rates than praziquantel monotherapy ( $\mathrm{RR}=1.25 ; 95 \% \mathrm{CI}$ : 1.09-1.37) in areas with intense transmission (moderate certainty of evidence) (Table 8) [49]. No significant adverse events were reported. 
Table 7. Accuracy of diagnostic tools for strongyloidiasis at different pre-test prevalence levels, 2012-February 2017.

\begin{tabular}{|c|c|c|c|c|c|c|c|c|c|c|c|c|c|}
\hline \multirow{2}{*}{$\begin{array}{c}\text { Index tests } \\
\text { Test } \% \text { Prevalence }^{b}\end{array}$} & \multicolumn{3}{|c|}{$\begin{array}{c}\text { True-Positives } \\
\text { Pre-Test Probability }\end{array}$} & \multicolumn{3}{|c|}{$\begin{array}{c}\text { False-Positives } \\
\text { Pre-Test Probability a }\end{array}$} & \multicolumn{3}{|c|}{$\begin{array}{c}\text { True-Negatives } \\
\text { Pre-Test Probability a }\end{array}$} & \multicolumn{3}{|c|}{$\begin{array}{c}\text { False-Negatives } \\
\text { Pre-Test Probability a }\end{array}$} & \multirow[t]{2}{*}{ \% Infected Correctly Diagnosed } \\
\hline & $2.5 \%$ & $10 \%$ & $30 \%$ & $2.5 \%$ & $10 \%$ & $30 \%$ & $2.5 \%$ & $10 \%$ & $30 \%$ & $2.5 \%$ & $10 \%$ & $30 \%$ & \\
\hline Baermann method [51] & 18 & 72 & 216 & 0 & 0 & 0 & 975 & 900 & 700 & 7 & 28 & 84 & $72 \%$ \\
\hline Agar plate [51] & 22 & 89 & 267 & 0 & 0 & 0 & 975 & 900 & 700 & 3 & 11 & 33 & $89 \%$ \\
\hline NIE-LIPS [62] & 21 & 85 & 255 & 49 & 45 & 35 & 926 & 855 & 665 & 4 & 15 & 45 & $85.1 \%$ \\
\hline IVD-ELISA (commercial test) [62] & 23 & 92 & 276 & 29 & 27 & 21 & 946 & 873 & 679 & 2 & 8 & 24 & $92 \%$ \\
\hline 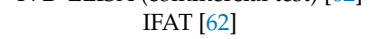 & 23 & 94 & 282 & 127 & 117 & 91 & 848 & 783 & 609 & 2 & 6 & 18 & $93.8 \%$ \\
\hline Bordier-ELISA (commercial kit) [62] & 23 & 91 & 272 & 58 & 54 & 42 & 917 & 846 & 658 & 2 & 9 & 28 & $90.7 \%$ \\
\hline SS-NIE-1 ELISA [63] & 24 & 95 & 285 & 68 & 63 & 49 & 907 & 837 & 651 & 1 & 5 & 15 & $95 \%$ \\
\hline
\end{tabular}

ELISA: enzyme-linked immunosorbent assay; IFAT: indirect fluorescent antibody technique; IVD: Invitro diagnostic test; LIPS: luciferase immunoprecipitation system; NIE: 31-kDa recombinant antigen from St. stercoralis.

a Data reported as effect per 1000 migrants tested.

pre-test prevalence or probability of having schistosomiasis in an at-risk population. 
Table 8. GRADE summary of findings of different schistosomiasis treatments vs. placebo, 2010-2016.

\begin{tabular}{|c|c|c|c|c|c|}
\hline \multirow{2}{*}{ Outcomes } & \multicolumn{2}{|c|}{$\begin{array}{l}\text { Anticipated Absolute Effects }{ }^{a} \\
(95 \% \mathrm{CI})\end{array}$} & \multirow{2}{*}{$\begin{array}{l}\text { Relative Chance of Cure } \\
\text { (95\% CI) }\end{array}$} & \multirow{2}{*}{$\begin{array}{c}\text { Number of } \\
\text { Participants/Studies }\end{array}$} & \multirow{2}{*}{$\begin{array}{l}\text { Certainty of the } \\
\text { Evidence (GRADE) }\end{array}$} \\
\hline & $\begin{array}{c}\text { Risk with Placebo per } \\
1000\end{array}$ & $\begin{array}{l}\text { Cure with Intervention } \\
\text { Drug }\end{array}$ & & & \\
\hline $\begin{array}{l}\text { Parasitological failure at } 1 \text { to } 2 \text { months (praziquantel } 40 \\
\mathrm{mg} / \mathrm{kg} \text { single dose) [48] }\end{array}$ & 908 & $381(263-562)$ & RR 0.42 (0.29 to 0.58$)$ & $864 / 7$ RCTs & High \\
\hline $\begin{array}{l}\text { Parasitological cure at } 1 \mathrm{month} \text { b }- \text { Sc. mansoni infections } \\
\text { (praziquantel } 40 \mathrm{mg} / \mathrm{kg} \text { single dose) [47] }\end{array}$ & 337 & $1000(347-1000)$ & RR 3.13 (1.03-9.53) & $414 / 2$ RCTs & Moderate $^{c}$ \\
\hline $\begin{array}{c}\text { Microhaematuria at } 8 \text { weeks (praziquantel } 40 \mathrm{mg} / \mathrm{kg} \\
\text { single dose) [48] }\end{array}$ & 281 & $149(93-236)$ & RR $0.53(0.33-0.84)$ & $119 / 1 \mathrm{RCT}$ & Low d,e,f \\
\hline $\begin{array}{l}\text { Infection rate of Sc. japonicum (artemether monotherapy } 6 \\
\mathrm{mg} / \mathrm{kg} \text { ) [49] }\end{array}$ & 175 & $44(28-70)$ & RR $0.25(0.16-0.40)$ & 8051/13 RCTs & Moderate $^{c}$ \\
\hline $\begin{array}{l}\text { Parasitological cure rate of Schistosoma species. } \\
\text { (Artesunate-monotherapy }(4 \mathrm{mg} / \mathrm{kg} \text { daily for three } \\
\text { consecutive days) }[49]\end{array}$ & $615^{*}$ & $302(172-459)$ & RR $0.49(0.28-0.75)$ & $800 / 7$ RCTS & Moderate $^{c}$ \\
\hline $\begin{array}{l}\text { Adverse events, minor (praziquantel } 40 \mathrm{mg} / \mathrm{kg} \text { single } \\
\text { dose) [49] }\end{array}$ & None & None & Not estimable & 1591/9 RCTs & Low $^{\mathrm{d}}$ \\
\hline
\end{tabular}

CI: confidence interval; GRADE: Grading of Recommendations, Assessment, Development and Evaluation; RR: risk ratio; RTC: randomized controlled trial. * praziquantel 40 mg/kg once.

The risk in the intervention group per 1000 persons treated $(95 \% \mathrm{CI})$ was based on the assumed risk in the comparison group and the relative effect of the intervention (and its $95 \% \mathrm{CI})$.

only Sc. mansoni infections reported.

Downgraded by 1 for indirectness: only two trials from limited settings evaluated this comparison.

The trial was under-powered; downgraded by 1.

Only a single trial reported this outcome.

Publication bias was unclear. 
Table 9. GRADE summary of findings on ivermectin $(200 \mathrm{mg} / \mathrm{kg}) \mathrm{vs}$. albendazole or thiabendazole for the treatment of strongyloidiasis, and certainty of evidence on treatment efficacy, benefits and harms, 2010-2016.

\begin{tabular}{|c|c|c|c|c|c|}
\hline \multirow[b]{2}{*}{ Outcomes } & \multicolumn{2}{|c|}{$\begin{array}{l}\text { Anticipated Absolute Effects } \\
(95 \% \text { CI) }\end{array}$} & \multirow{2}{*}{$\begin{array}{l}\text { Relative Chance of Cure } \\
\qquad\left(95 \% \text { CI) }{ }^{b}\right.\end{array}$} & \multirow{2}{*}{$\begin{array}{c}\text { Number of } \\
\text { Participants/Studies }\end{array}$} & \multirow{2}{*}{$\begin{array}{l}\text { Certainty of the } \\
\text { Evidence (GRADE }\end{array}$} \\
\hline & $\begin{array}{l}\text { Cure with Comparator } \\
\text { Drug per } 1000^{a}\end{array}$ & $\begin{array}{l}\text { Cure with Intervention } \\
\text { Drug-Ivermectin } \\
(200 \mathrm{mg} / \mathrm{kg})^{b}\end{array}$ & & & \\
\hline Cure overall assessed at 5 weeks—albendazole [52] & 480 & $\begin{array}{c}840 \\
(720-980)\end{array}$ & $\begin{array}{c}\text { RR 1.79 } \\
(1.55-2.08)\end{array}$ & $478 / 4$ RCTs & Moderate ${ }^{\mathrm{d}}$ \\
\hline Adverse events assessed at 5 weeks_-albendazole [52] & 260 & $\begin{array}{c}210 \\
(150-290)\end{array}$ & $\begin{array}{l}\text { RR } 0.80 \\
(0.59-1.09)\end{array}$ & $518 / 4$ RCTs & Low ${ }^{c, g}$ \\
\hline Cure overall assessed at 11 weeks_-thiabendazole [52] & 690 & $\begin{array}{c}740 \\
(660-820)\end{array}$ & $\begin{array}{l}\text { RR } 1.07 \\
(0.96-1.20)\end{array}$ & $467 / 3 \mathrm{RCTs}$ & Moderate ${ }^{\mathrm{e}}$ \\
\hline Adverse events assessed at 11 weeks_-thiabendazole [52] & 730 & $\begin{array}{c}230 \\
(150-360)\end{array}$ & $\begin{array}{l}\text { RR } 0.31 \\
(0.20-0.50)\end{array}$ & $507 / 3 \mathrm{RCTs}$ & Moderate ${ }^{f}$ \\
\hline
\end{tabular}

PICO—Patient or population: sersons with Strongyloides stercoralis infection; Setting: south-east-Asia, America and Europe; Intervention: ivermectin; Comparison: albendazole and thiabendazole. CI: confidence interval; GRADE: Grading of Recommendations, Assessment, Development and Evaluation; RR: risk ratio; RTC: randomized controlled trial.

a Albendazole or thiabendazole.

b The risk in the intervention group per 1000 persons treated $(95 \% \mathrm{CI})$ was based on the assumed risk in the comparison group and the relative effect of the intervention (and its $95 \% \mathrm{CI})$

c No method of allocation concealment in two trials and no method of allocation described.

Two trials did not conceal allocation and no method of allocation was described.

Two trials did not conceal allocation and no method of allocation was described in one trial.

Two trials did not conceal allocation and no method of allocation was described.

$\mathrm{g} \quad$ Wide range of estimates in three trials could include substantive fewer events. 
Only one systematic review was included which addressed the efficacy of ivermectin vs. albendazole or thiabendazole for treating chronic strongyloidiasis infection (Table 9) [52]. Parasitological cure determined with both serological and conventional techniques was higher with ivermectin (single-/double-dose) treatment than with albendazole $84 \%$ vs. $48 \%$ (RR $=1.79 ; 95 \%$ CI: 1.55-2.08) (moderate-quality evidence) [52]. When ivermectin was compared with thiabendazole, there was no distinction in parasitological cure, i.e., $74 \%$ vs. $68 \%(R R=1.07 ; 95 \%$ CI: $0.96-1.2)$, but adverse events were less frequent with ivermectin ( $R R=0.31 ; 95 \%$ CI: $0.20-0.50)$ than with thiabendazole [52] (moderate certainty of evidence). No serious adverse events or deaths were reported with either ivermectin or thiabendazole.

\subsection{Resource use, Costs and Cost-Effectiveness}

\subsubsection{Strongyloidiasis}

Three economic studies of moderate quality support a strategy of presumptive treatment for strongyloidiasis in migrants from high-risk backgrounds [66-68]. One study showed potential cost savings of universal treatment with albendazole compared with i) no intervention (watchful waiting); and compared with ii) universal stool-based screening; in migrant populations in the U.S. [66]. Sensitivity analyses indicated a best-case scenario of large savings from presumptive treatment, and a worst-case scenario in which treatment was still cost effective at the $\$ 30,000 /$ QALY threshold (1997 U.S. dollars).

The second study on presumptive treatment for strongyloidiasis in migrants living in the U.S. in California and New York compared: i) presumptive treatment with albendazole for 3 or 5 days; ii) presumptive treatment with one dose of ivermectin; iii) treatment in those with documented eosinophilia; and iv) no intervention [67]. It indicated that presumptive treatment with ivermectin was cost-effective at a threshold of less than USD 10,000 (EUR 9667) per QALY across a range of prevalence values in migrants living in the U.S. [67]. This study did not include antibody detection among the diagnostic tools. At a prevalence higher than 10\%, treatment with ivermectin cost less than USD 2000 (EUR 1983) per QALY. These results were robust across a wide range of sensitivity analyses [67].

The third more recent study on presumptive treatment for hookworm and strongyloidiasis in U.S.-bound Asian populations indicated that treatment in the destination country with albendazole and ivermectin was likely to be cost-effective relative to no screening or screening and treatment strategies in the country of origin among refugees from high-prevalence countries [68]. For strongyloidiasis, overseas treatment cost less than USD 40,000 (EUR 31,092) per QALY gained at prevalence greater than $1 \%$ and fell to less than USD 18,000 (EUR 13,991) per QALY gained at prevalence greater than 3\%.

\subsubsection{Schistosomiasis}

There were no cost-effectiveness studies of screening and presumptive treatment in migrants at risk of schistosomiasis. In non-migrant populations, a recent costing study compared the costs of single and double KK tests with a urine dipstick test [69] for Sc. haematobium diagnosis in areas of high endemicity. The results of this preliminary costing study indicated similar costs of around USD 6-7 (EUR 5-6) per test for single KK stool and urine tests; however, the quality of evidence for resource use was low. A cost-effectiveness study by King et al. compared single-dose ( $40 \mathrm{mg} / \mathrm{kg}$ body weight) and double-dose ( $40 \mathrm{mg} / \mathrm{kg}$ doses separated by $2-8$ weeks) presumptive treatment with praziquantel for schistosomiasis in high-prevalence $(>40 \%)$ settings in Africa [65]. Double-dose praziquantel was found deemed to be highly cost-effective (ICER of less than USD 500 (EUR 471)/QALY) compared with single-dose treatment.

\section{Discussion}

The rationale for screening for strongyloidiasis and schistosomiasis in the EU/EEA and not other parasitic infections is based on the estimated prevalence of these parasitic infections among migrants 
from endemic countries; potential prevention of fatal complications through early case detection and treatment, and secondary transmission in asymptomatic patients based on a highly sensitive test and very effective and safe treatment $[11,35,36,71]$. Therefore, the implementation of a screening programme would allow early detection of the infection in individuals at risk, before they develop a severe condition which may justify the screening itself.

Although quality data on the prevalence of schistosomiasis and strongyloidiasis among migrant populations in the EU/EEA is limited, available data from endemic regions shows that prevalence of schistosomiasis is between $20 \%$ and $40 \%$ and prevalence of strongyloidiasis is between $10 \%$ and $40 \%$ [3-5]. However, there is a rationale for public health surveillance for schistosomiasis and strongyloidiasis to inform proper surveillance of mobile population from the regions [30]

Overall, systematic reviews showed that antibody-detecting serological tests are the most effective screening tests for detection of schistosomiasis and strongyloidiasis in low-endemicity settings, because they have higher sensitivity than conventional parasitological methods $[19,44,45,50,53]$. Newer serological tests were shown to be more effective than conventional techniques such as agar plate culture and the Baermann method for strongyloidiasis and KK for Sc. mansoni. These conventional techniques, as well as PCR, failed to detect infections of very low intensity [64] although they were more specific than serological techniques [51,54]. They are also labor-intensive and require skilled personnel and are therefore not recommended as the first option for screening [19]. In contrast, serological testing is easier to perform in health facilities than collecting and testing faecal samples and can also be combined with other infectious disease screening tests.

One limitation of antibody-detecting serological tests, particularly with schistosomiasis, is that they cannot differentiate current from past infections; however, with strongyloidiasis, antibody titres decline after treatment over time in most patients [62,72]. In addition, in immuno-compromised patients, the sensitivity of serological tests may be reduced, and other additional screening methods may be needed if serology is negative. In this regard, the utility of PCR assay as an alternative screening method in immunosuppressed patients deserves further investigation.

Specifically, for Schistosoma spp. infections, available evidence shows that the IgM-ELISA [57], IHA [46] and ICT IgG-IgM [61] tests were the most effective screening tests in low-endemicity countries. In some low endemicity settings, two serological tests are performed, and a case is considered to be positive if either test is positive; in others, a combination of ELISA testing and KK faecal examinations is used to improve the accuracy of detection. However, Beltrame et al. advocate the use of the ICT IgG-IgM test as a single screening test (negative predictive value $>97 \%$ ) [61].

For strongyloidiasis, available evidence (of very low to low quality) shows that antibody-detecting blood tests using a variety of antigen preparations have a better detection rate than conventional parasitological methods, with IVD-ELISA, Bordier-ELISA and NIE LIPS being the most accurate tests [62]. Limitations of these serological tests include the large number of infective larvae required, cross-reactions with other nematode infections and lower sensitivity in immuno-compromised patients [19,62]. New tests based on the recombinant antigen Ss-NIE-1, although slightly less sensitive, but currently considerably more expensive than other serological techniques, show excellent specificity $[62,63]$ and, although not widely available, they may be useful when designing rapid tests [63].

For treatment of schistosomiasis, single-dose praziquantel is the drug of choice. Evidence from systematic reviews shows that treatment with praziquantel significantly increased parasitological cure and, achieved marked reductions in microhaematuria compared with placebo; praziquantel also has a very good safety profile $[47,48]$. For treatment of strongyloidiasis, there is evidence (of low to moderate quality) that ivermectin is more effective than albendazole [52] and evidence (of moderate quality) that ivermectin is as effective as thiabendazole, but much better tolerated; no difference in the efficacy of ivermectin was observed between endemic and non-endemic populations [52]. However, there are no studies on the potential harms of large-scale administration of ivermectin (although widespread experience with filariasis control is reassuring). 
Implementing presumptive treatment either with ivermectin or praziquantel requires additional complex screening strategies to identify individuals with loiasis or neurocysticercosis for whom these drugs might be inappropriate [70,71] and recently published recommendations specify that immigrants arriving from endemic areas should undergo a thorough clinical screening before being given either praziquantel or albendazole [73]. In addition, ivermectin is not readily available in most endemic and non-endemic countries and has limited approval by regulatory authorities in the EU/EEA.

We found no studies evaluating the cost-effectiveness of schistosomiasis screening and treatment interventions in migrant populations. For schistosomiasis, no studies were available on the cost of screening tests based on antibody detection in the non-endemic setting. In endemic settings, double-dose praziquantel was deemed to be highly cost-effective compared with a single dose and was considered robust to plausible changes in parameter estimates [65]. Further economic studies are required to provide better data on the cost-effectiveness of a test-and-treat strategy for schistosomiasis in non-endemic countries. For strongyloidiasis, three studies indicated that presumptive treatment with albendazole or ivermectin was cost-saving or cost-effective, in migrants to the U.S. or in endemic settings [66-68]. The limitations of these studies may decrease the relevance of the results for migrant populations in the EU/EEA. Most of the economic studies identified were limited to Asian populations and not based on screening with antibody testing in a non-endemic setting. However, where the prevalence of schistosomiasis and strongyloidiasis is greater than $1 \%$ and the price of presumptive treatment is similar to that used in the economic evaluations identified in this review, presumptive treatment with ivermectin or albendazole is likely to be cost-effective for migrants to the EU/EEA.

The strengths of our study include the use of the GRADE methodology to evaluate the quality and strength of the evidence and effect size in the included studies. The primary outcomes-parasitological cure or failure for efficacy of treatment and accuracy for screening-were objective measures. The individual studies in the included systematic reviews originated from different regions and countries with moderate to high endemicity for both parasites, increasing the generalizability of the results.

We did not identify any systematic reviews or RCTs on screening for schistosomiasis and strongyloidiasis in newly arrived migrants to EU/EEA. RCTs on preventive screening are rare, and so we used a logic model approach, as recommended at US Task Force on Preventive Health Care, and present data on population prevalence, diagnostic accuracy, treatment effectiveness and cost-effectiveness [70,74]. Other limitations include the lack of accurate data on the prevalence of schistosomiasis and strongyloidiasis among migrants from endemic countries entering the EU/EEA and the lack of data on the cost-effectiveness of screening and treating migrants for these parasitic infections. Further studies evaluating the effectiveness and cost-effectiveness of screening intervention in migrant populations are warranted.

The results of this systematic review indicate that although the certainty of desirable over undesirable effects of screening mobile and high-risk migrant populations from endemic areas is low to moderate, there is a rationale for screening, particularly in immunosuppressed patients since there is a high value placed on uncertain but potentially life-preserving benefits as suggested elsewhere [75]. Both schistosomiasis and strongyloidiasis can become chronic and cause severe long-term complications if untreated and the health benefits of intervention therefore outweigh its potential harms. Effective diagnostic tests are available and treatments for both infections are efficacious, well tolerated and safe with few exceptions [48,52,54,62].

Presumptive single-dose therapy of strongyloidiasis with ivermectin for all migrants is likely to be cost-effective; however, the feasibility of this measure has not been demonstrated in clinical studies in non-endemic settings. Importantly, implementing presumptive treatment either with ivermectin for strongyloidiasis or praziquantel for schistosomiasis requires additional screening strategies to identify individuals for whom these drugs might be harmful.

The evidence suggest screening should target people arriving from endemic areas, but national screening strategies will need to be tailored to the specific context of individual EU/EEA countries 
and, in particular, the countries of origin of migrants to those countries. Although, there are no studies on the extent to which multiple screening tests for infectious diseases in migrants can improve cost-effectiveness, integrating innovative public health screening strategies for schistosomiasis and strongyloidiasis with other infectious diseases will improve surveillance data as well as reduce costs.

However, the optimal approach to delivery of screening will need to consider a global perspective, as well as depend on the health system context in individual EU/EEA countries. In this regard, addressing lack of access to healthcare for migrants, heterogeneity of screening strategies applicable in member states, and improving health professionals' knowledge and training of migrant related infectious diseases should improve the responsiveness of the public health care system with regards to coverage and uptake of screening at the level of primary health care.

Finally, although we consider that sufficient evidence exists to justify screening for strongyloidiasis and schistosomiasis immigrants coming to the EU/EEA from endemic areas, further assessment of the benefits and risks of screening and treatment is needed. More specifically, additional economic analysis is required, in particular to evaluate the costs of a test and treat strategy and to compare the cost-effectiveness of screening and of presumptive treatment.

\section{Conclusions}

This systematic review provides a compendium of indirect evidence that support the screening for strongyloidiasis and schistosomiasis in migrants coming from endemic areas to the EU/EEA, and particularly in immunosuppressed or at-risk-of immunosuppression patients.

Screening for strongyloidiasis and schistosomiasis should be considered based on serological testing in the absence of immunosuppression. Ivermectin and praziquantel have demonstrated a high efficacy, an excellent safety profile, and a potentially easy schedule for the treatment of strongyloidiasis and schistosomiasis. Economic modelling suggests presumptive single-dose treatment of strongyloidiasis with ivermectin for all migrants is likely cost-effective, but the feasibility of this strategy has yet to be demonstrated in clinical studies in non-endemic settings.

Author Contributions: Conceptualization, E.N.A., A.R.-M., P.A.-C., C.G. and K.P.; Methodology, E.N.A., A.R.-M., E.B., P.A.-C., R.L.M. and K.P.; Software, E.N.A., D.T.M., I.A.-R. and K.P.; Validation, E.N.A., A.T., D.T.M., I.A.-R., N.M. and Z.B.; Formal Analysis, E.N.A., N.M., I.A.-R., R.L.M., A.T., N.R. and Z.B.; Investigation, E.N.A., N.M., T.N., E.B., Z.B., A.R.-M. and R.L.M.; Resources, E.N.A., I.A.-R., T.N., P.A.-C. and K.P.; Data Curation, E.N.A., D.T.M., and I.A.-R.; Writing-Original Draft Preparation, E.N.A., A.R.-M., P.A.-C., R.L.M., and K.P.; Writing-Review \& Editing, E.N.A., E.B., A.R.-M., P.A.-C., R.L.M., T.N., and K.P.; Visualization, E.N.A., A.R.-M., P.A.-C., R.L.M. and Z.B.; Supervision, E.N.A., A.R.-M., P.A.-C., R.L.M., T.N. and K.P.; Project Administration, E.N.A., A.R.-M., P.A.-C., T.N. and K.P.; Funding Acquisition, K.P., T.N., and P.A.-C.

Funding: European Health Group and the European Centre for Disease Prevention and Control (ECDC); FWC No ECDC/2015/016; Specific Contract No 1 ECD.5748. The ECDC has suggested experts for review working groups, requested progress reports and provided stakeholder feedback on the proposed protocols. The Barcelona Institute for Global Health (ISGlobal) Research group was supported by Agència de Gestió d'Ajuts Universitaris i de Recerca (AGAUR) (2014SGR26). The views expressed in this publication are those of the author(s) and not necessarily those of the Barcelona Institute for Global Health (ISGlobal) Research group.

Acknowledgments: We appreciate the contributions of Alain Mayhew, Research Associate, C.T. Lamont Primary Health Care Research Centre, Bruyère Research Institute, who facilitated the disease group meeting as well as offered technical guidance to the IP team. Eric N. Agbata is a doctoral candidate for the PhD in Methodology of Biomedical Research and Public Health (Department of Paediatrics, Obstetrics, Gynaecology and Preventive Medicine), Universidad Autònoma de Barcelona, Barcelona, Spain. His significant contributions to this project will form part of his thesis.

Conflicts of Interest: The authors declare no conflict of interest. 
Appendix A. Logic Model-Analytic Framework for Screening and Treatment for Schistosomiasis and Strongyloidiasis in Migrants

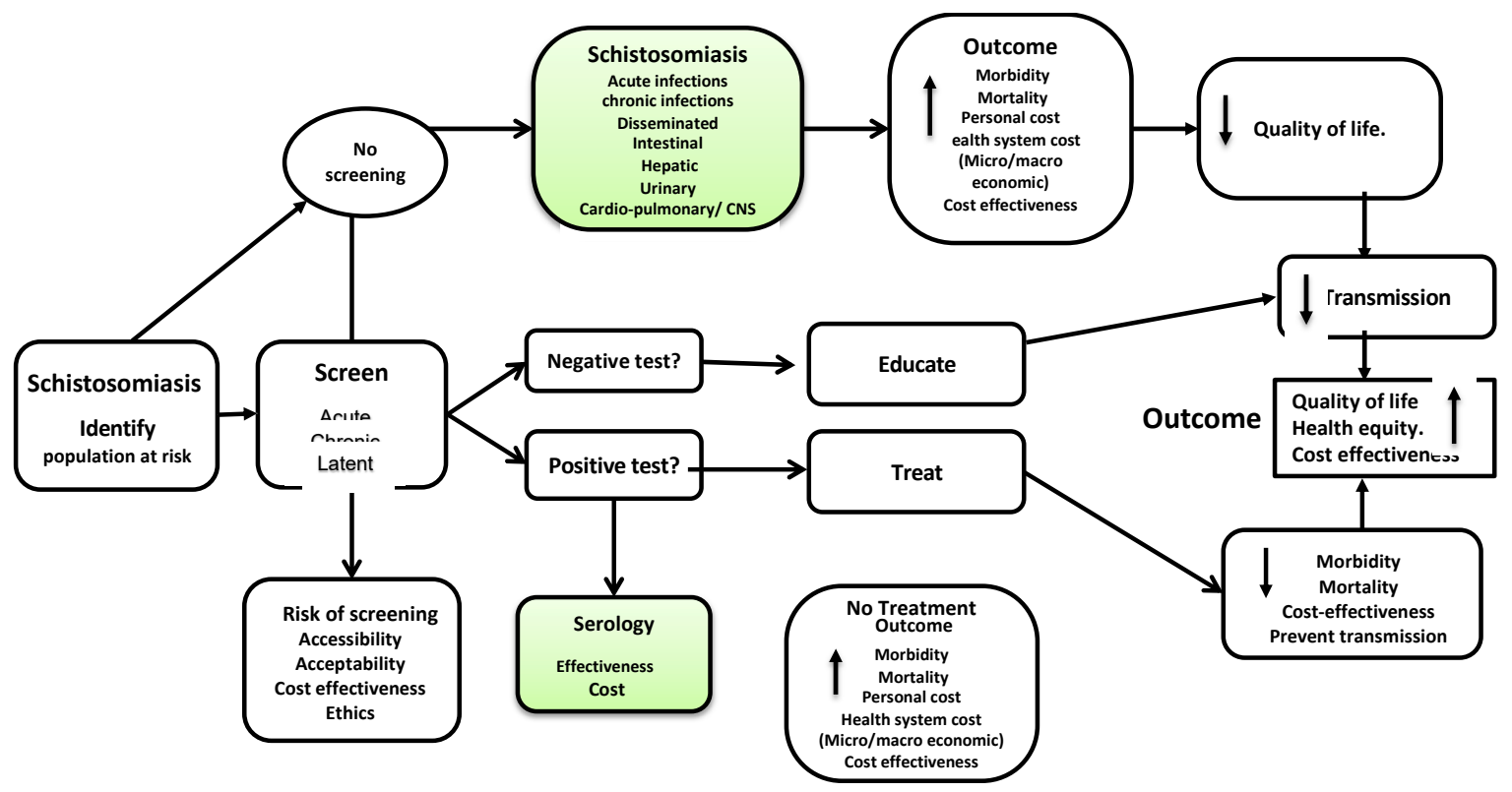

Figure A1. Analytic framework for screening and treatment of schistosomiasis in migrants.

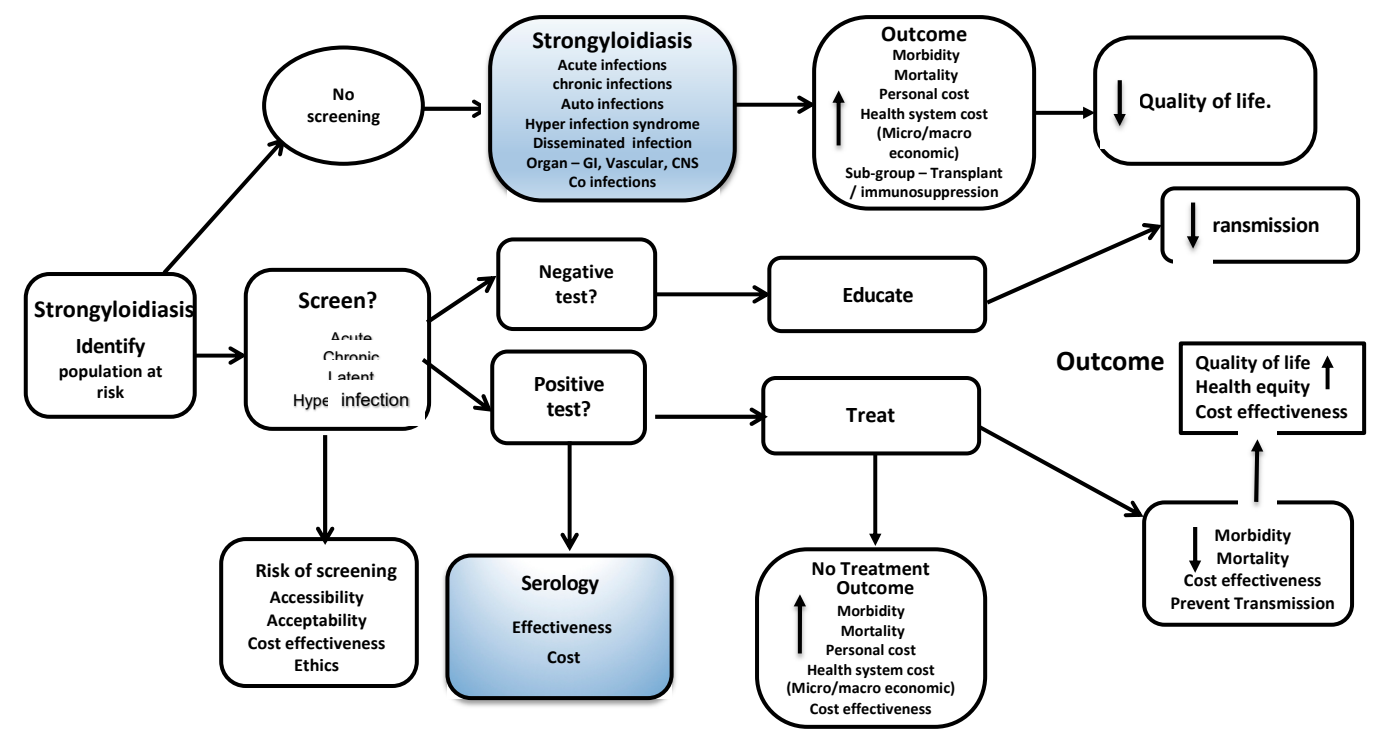

Figure A2. Analytic framework for screening and treatment of strongyloidiasis in migrants.

\section{Appendix B. List of Sites and Literature Search Strategy}

\section{Literature search strategy for systematic review}

The used search strategies for the identification of systematic reviews are listed here.

A. Database: Ovid MEDLINE(R) 1946 to Present with Daily Update

Search Date: 15 April 2016

1. $\quad \exp$ Schistosoma/ (15595)

2. bilharzia\$.tw. (2431)

3. $\quad \exp$ Schistosomiasis/ (21432) 
4. schistosom\$.tw. (25367)

5. katayama fever\$.tw. (30)

6. or /1-5 (30014)

7. Strongyloides/ (985)

8. Strongyloides stercoralis/ (1044)

9. Strongyloidiasis/ (3301)

10. strongyloid\$.tw. (3988)

11. or /7-10 (4959)

12. 6 or $11(34621)$

13. exp Mass Screening/ (107821)

14. (screened or screening?) tw. (417896)

15. Early Diagnosis/ (19041)

16. (detected or detection? or diagnos $\$$ or discover $\$$ or indentif\$) tw. (2972048)

17. exp Population Surveillance/ (56090)

18. (disease? adj2 surveillance) tw. (4053)

19. Contact Tracing/ (3521)

20. contact tracing tw. (1152)

21. or $/ 13-20(3301561)$

22. meta analysis $\mathrm{mp}$, pt. (91365)

23. review pt. (2035657)

24. search\$ tw. (253765)

25. or / 22-24 (2222329)

26. animals / not (humans / and animals/) (4194238)

27. 25 not 26 (2065589)

28. 12 and 21 and $27(711)$

29. 28 and ( $2010 \$$ or $2011 \$$ or $2012 \$$ or $2013 \$$ or $2014 \$$ or $2015 \$$ or $2016 \$$ ) ed. (222)

30. remove duplicates from 29 (218)

B. Database: Embase <1980 to 2016 April 14> Search Date: 15 April 2016

1. $\operatorname{exp~Schistosoma/~(19846)~}$

2. bilharzia\$.tw. (2115)

3. exp schistosomiasis/ (20241)

4. schistosom\$.tw. (26744)

5. katayama fever\$.tw. (40)

6. or /1-5 (33204)

7. Strongyloides/ (1220)

8. Strongyloides stercoralis/ (2315)

9. strongyloidiasis/ (3835)

10. strongyloid\$.tw. (4704)

11. or /7-10 (6600)

12. 6 or $11(39071)$

13. exp mass screening/ (178654)

14. (screened or screening?).tw. (614882)

15. early diagnosis/ (82347) 
16. parasite identification/ (13161)

17. ((case? or early or parasit\$) adj5 (detected or detection? or diagnos $\$$ or discover $\$$ or egg or indentif\$)).tw. (385884)

18. exp health survey/ (182738)

19. (disease? adj2 surveillance).tw. (5156)

20. contact examination/ (2830)

21. contact tracing.tw. (1448)

22. or/13-21 (1237076)

23. meta analys\$.mp. (167508)

24. search\$.tw. (362044)

25. review.pt. (2131214)

26. or $/ 23-25(2472677)$

27. (exp animal/ or animal.hw. or nonhuman/) not (exp human/ or human cell/ or (human or humans) ti.) (5499319)

28. 26 not $27(2251777)$

29. 12 and 22 and 28 (455)

30. 29 and ( $2010 \$$ or $2011 \$$ or $2012 \$$ or $2013 \$$ or $2014 \$$ or $2015 \$$ or $2016 \$)$ dd. (195)

31. remove duplicates from 30 (190)

C. Database: EBSCO CINAHL <1970 to April 2016>

Search Date: 15 April 2016

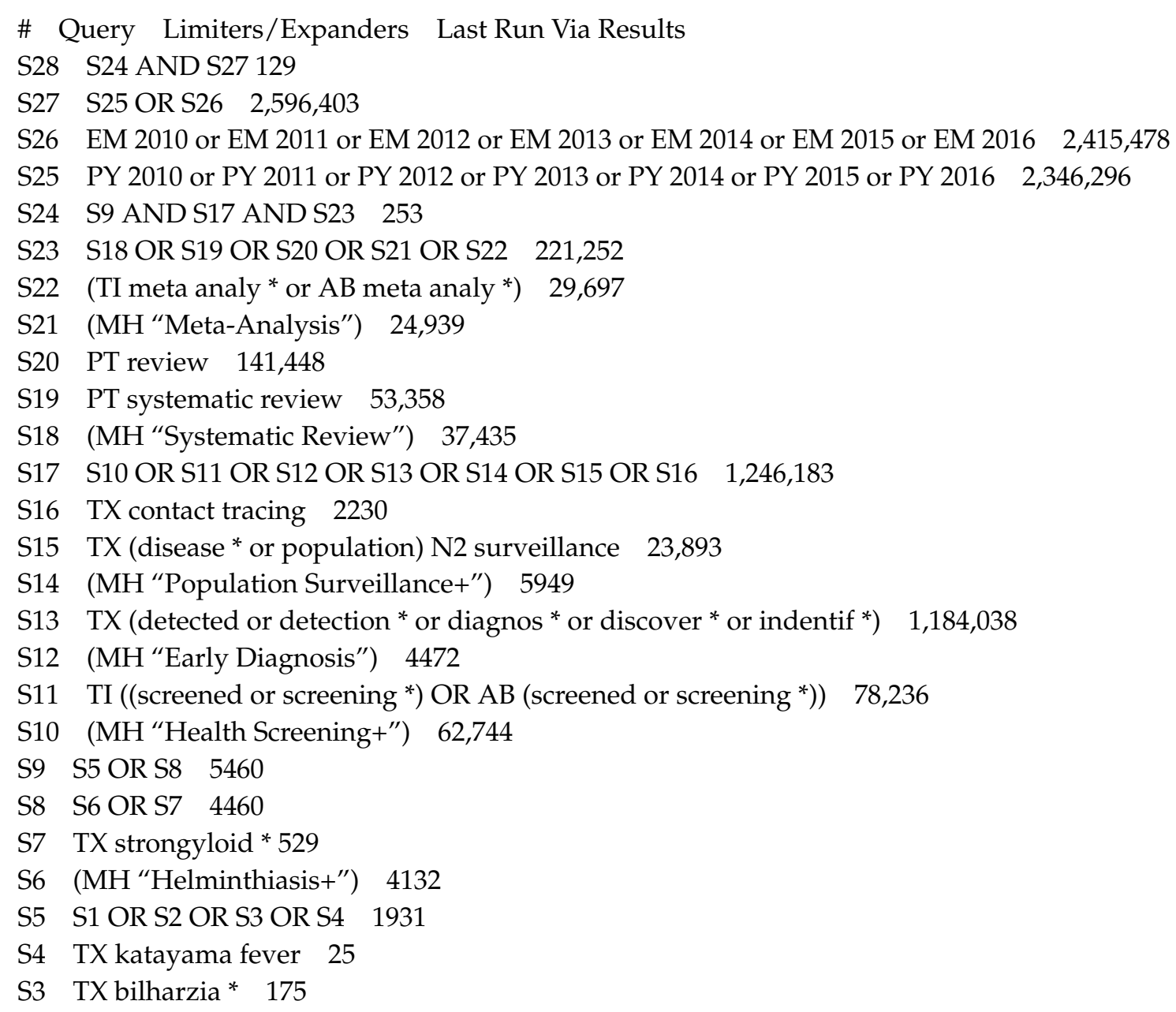


S2 TX schistosome* 1871

S1 (MH "Schistosomiasis+") 756

$* * * * * * * * * * * * * * * * * * * * * * * * * * *$

D. Databases: Database of Abstracts of Reviews of Effects (DARE) and Cochrane Database of Systematic Reviews (CDSR)

Search Date: 15 April 2016

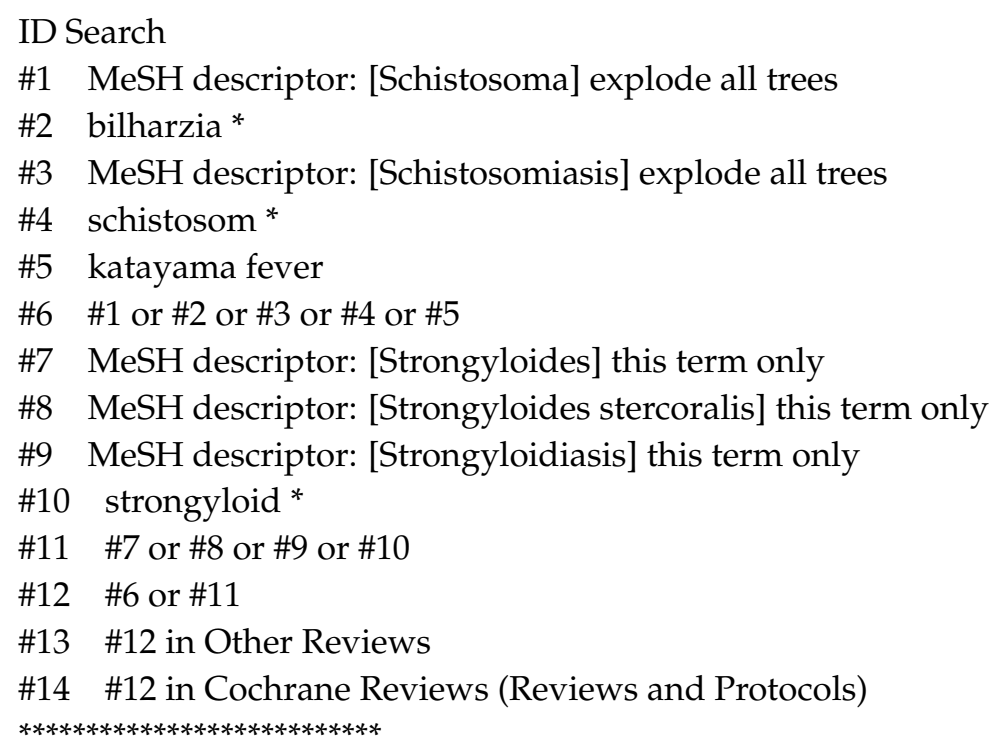

\section{Literature search strategy for systematic search for cost-effectiveness studies}

The used search strategies for the identification of systematic reviews on cost-effectiveness are listed here.

A. Database: Ovid MEDLINE(R) Epub Ahead of Print <May Week 3 2016>, Ovid MEDLINE(R) 1946 to Present with Daily Update

Search Date: 31 May 2016

1. exp Schistosoma/ (15714)

2. bilharzia\$.tw. (2438)

3. exp Schistosomiasis / (21583)

4. schistosom\$.tw. (25722)

5. katayama fever\$.tw. (30)

6. or $/ 1-5(30381)$

7. Strongyloides/ (990)

8. Strongyloides stercoralis/ (1056)

9. Strongyloidiasis/ (3319)

10. strongyloid\$.tw. (4079)

11. or/7-10 (5051)

12. 6 or $11(35067)$

13. exp Mass Screening/ (108535)

14. (screened or screening? or tested or testing or tests).tw. (1734474)

15. Early Diagnosis/ (19350)

16. (detected or detection? or diagnos $\$$ or discover $\$$ or indentif $\$$ ).tw. (3053822)

17. exp Population Surveillance/ (56687)

18. (disease? adj2 surveillance).tw. (4195) 
19. Contact Tracing/ (3563)

20. contact tracing.tw. (1176)

21. or $/ 13-20(4387118)$

22. meta analysis.mp,pt. (96759)

23. review.pt. (2060867)

24. search\$.tw. (266775)

25. guideline.pt. (15780)

26. guideline/ (15780)

27. guidelines as topic/ (34071)

28. practice guideline.pt. (21216)

29. practice guideline/ (21216)

30. practice guidelines as topic/ (91792)

31. (CPG or CPGs or guidance or guideline? or recommend\$ or standard?).ti. (147179)

32. exp clinical pathway/ (5273)

33. exp clinical protocol/ (139345)

34. ((care or clinical) adj2 pathway?).tw. (5129)

35. or / 22-34 (2572065)

36. 12 and 21 and 35 (960)

37. animals/ not (humans/ and animals/) (4215704)

38. 36 not $37(838)$

39. 38 and ( $2010 \$$ or $2011 \$$ or $2012 \$$ or $2013 \$$ or $2014 \$$ or $2015 \$$ or $2016 \$)$.ed. (271)

40. remove duplicates from 39 [reviews and guidelines] (261)

41. exp "costs and cost analysis" / (197942)

42. cost\$.mp. (467877)

43. cost effective\$.tw. (83090)

44. cost benefit analys\$.mp. (67319)

45. health care costs.mp. (37157)

46. or /41-45 (477217)

47. 12 and 21 and 46 (260)

48. animals/ not (humans/ and animals/) (4215704)

49. 47 not 48 (222)

50. 49 and ( $2010 \$$ or $2011 \$$ or $2012 \$$ or $2013 \$$ or $2014 \$$ or $2015 \$$ or $2016 \$)$.ed. (82)

51. remove duplicates from 50 (78)

B. Database: Embase <1974 to 2016 Week 22>

Search Date: 31 May 2016

1. exp Schistosoma/ (21727)

2. bilharzia\$.tw. (2492)

3. exp schistosomiasis/ (21930)

4. $\quad$ schistosom\$.tw. (29047)

5. katayama fever\$.tw. (42)

6. or/1-5 (36157)

7. Strongyloides/ (1229)

8. Strongyloides stercoralis/ (2447)

9. strongyloidiasis/ (3986)

10. strongyloid\$.tw. (4977) 
11. or $/ 7-10(6962)$

12. 6 or $11(42352)$

13. exp mass screening/ (182895)

14. (screened or screening? or tested or testing or tests).tw. (2429856)

15. early diagnosis/ (83110)

16. parasite identification/ (13222)

17. ((case? or early or parasit\$) adj5 (detected or detection? or diagnos\$ or discover $\$$ or egg or indentif\$)).tw. (405389)

18. exp health survey/ (184236)

19. (disease? adj2 surveillance).tw. (5253)

20. contact examination/ (2867)

21. contact tracing.tw. (1512)

22. or/13-21 (2999272)

23. meta analys\$.mp. (170914)

24. search\$.tw. (371898)

25. review.pt. (2163187)

26. guideline.pt. (0)

27. guideline/ (144)

28. guidelines as topic/ (229895)

29. practice guideline.pt. (0)

30. practice guideline/ (275502)

31. practice guidelines as topic/ (171091)

32. (CPG or CPGs or guidance or guideline? or recommend\$ or standard?).ti. (203285)

33. exp clinical pathway/ (6983)

34. exp clinical protocol/ (75932)

35. ((care or clinical) adj2 pathway?).tw. (9455)

36. $\quad$ or $/ 23-35$ (2900847)

37. 12 and 22 and $36(824)$

38. (exp animal/ or animal.hw. or nonhuman/) not (exp human/ or human cell/ or (human or humans).ti.) (5865460)

39. 37 not $38(678)$

40. 39 and $(2010 \$$ or $2011 \$$ or $2012 \$$ or $2013 \$$ or $2014 \$$ or $2015 \$$ or $2016 \$)$.dd. (304)

41. remove duplicates from 40 [reviews and guidelines] (295)

42. cost effectiveness analysis / (114264)

43. cost.tw. (387431)

44. costs.tw. (208732)

45. or $/ 42-44(544771)$

46. 12 and 22 and $45(274)$

47. (exp animal/ or animal.hw. or nonhuman/) not (exp human/ or human cell/ or (human or humans).ti.) (5865460)

48. $46 \operatorname{not} 47(223)$

49. 48 and ( $2010 \$$ or $2011 \$$ or $2012 \$$ or $2013 \$$ or $2014 \$$ or $2015 \$$ or $2016 \$) . d d$. (115)

50. remove duplicates from 49 [costing] (111)

C. Databases: Database of Abstracts of Reviews of Effects (DARE) and Cochrane Database of Systematic Reviews (CDSR) and NHS EED

Search Date: 31 May 2016 
ID Search

\#1 MeSH descriptor: [Schistosoma] explode all trees

\#2 bilharzia*

\#3 MeSH descriptor: [Schistosomiasis] explode all trees

\#4 schistosom*

\#5 katayama fever

\#6 \#1 or \#2 or \#3 or \#4 or \#5

\#7 MeSH descriptor: [Strongyloides] this term only

\#8 MeSH descriptor: [Strongyloides stercoralis] this term only

\#9 MeSH descriptor: [Strongyloidiasis] this term only

$\# 10$ strongyloid*

\#11 \#7 or \#8 or \#9 or \#10

\#12 \#6 or \#11

\#13 \#12 in Other Reviews

\#14 \#12 in Cochrane Reviews (Reviews and Protocols)

\#15 \#12 in Economic Evaluations

$* * * * * * * * * * * * * * * * * * * * * * * * * *$

D. Database: EBSCO CINAHL <1970 to May 2016>

Search Date: 31 May 2016

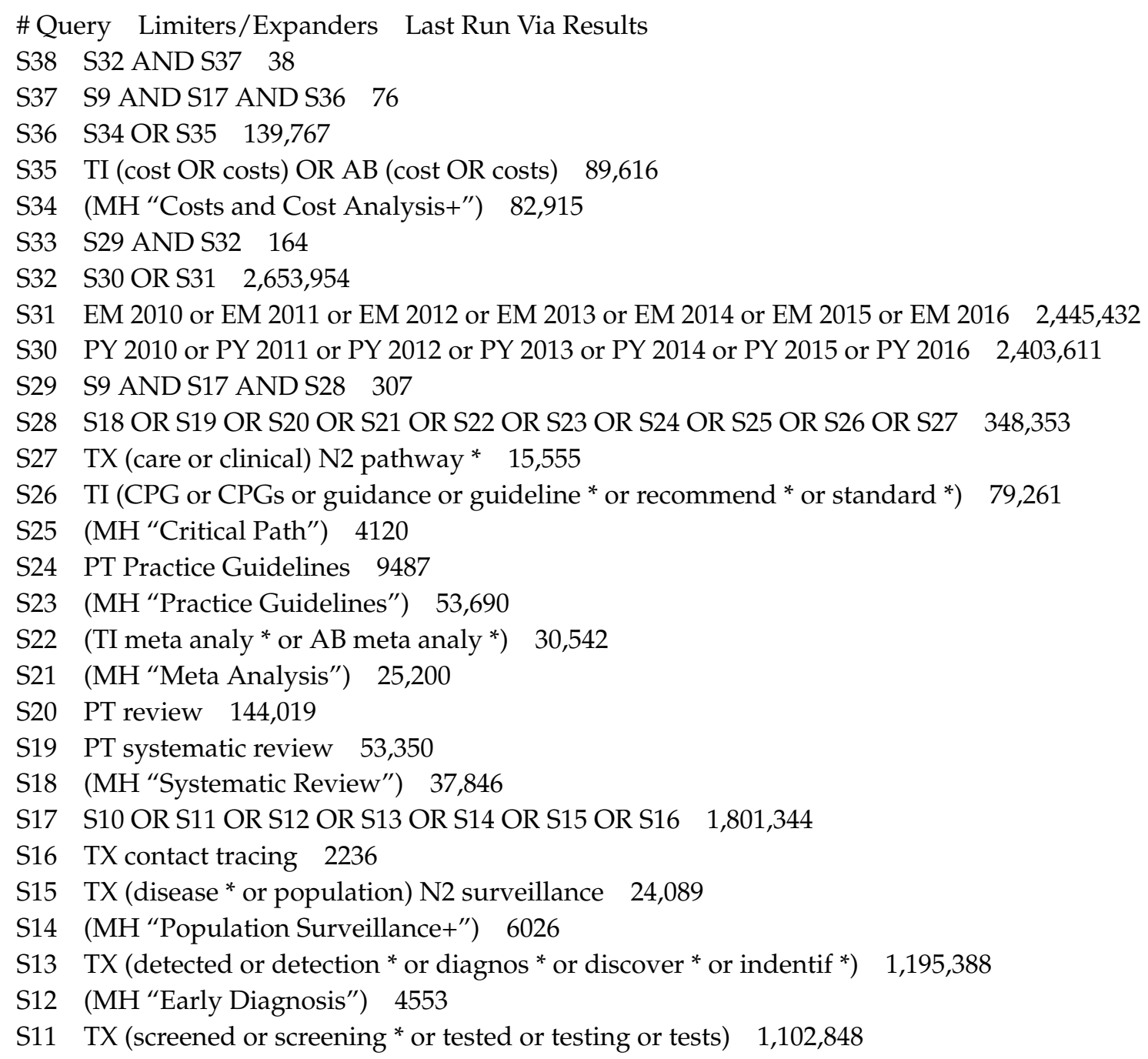




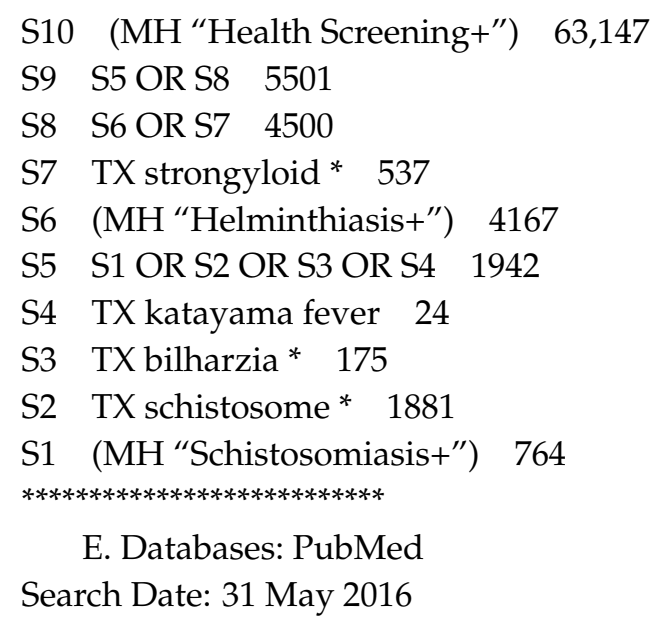

$((((((($ schistosome * or bilharzia * or katayama or strongyloid *))) AND ((screened or screening * or tested or testing or tests $))))$ AND (((CPG or CPGs or guidance or guideline * or metaanalysis or meta-analysis or recommend * or review or standard or standards)))) AND ((publisher [3]))))) (8)

$((((((($ schistosome * or bilharzia * or katayama or strongyloid *))) AND ((screened or screening * or tested or testing or tests)))) AND (((cost or costs)))) AND ((publisher [3]))))) (2)

$* * * * * * * * * * * * * * * * * * * * * * * * * * *$

3. Update Literature strategy for primary studies on diagnostic or screening tools for schistosomiasis.

A. Database: Ovid MEDLINE(R)—1946 to February 2017.

1. Schistosomiasis/ (13485)

2. Schistosomiasis.mp. (24533)

3. snail fever.mp. (10)

4. schistosome *.mp. (5528)

5. $\quad$ exp "Sensitivity and Specificity" / (495027)

6. $\quad$ sensitivity.tw. (638974)

7. $\quad$ specificity.tw. (379605)

8. ((pre-test or pretest) adj probability).tw. (1695)

9. post-test probability.tw. (441)

10. predictive value\$.tw. (85102)

11. likelihood ratio\$.tw. (11639)

12. or $/ 5-11(1217873)$

13. or $/ 1-4(26340)$

14. 12 and 13 (1493)

15. limit 14 to humans (1112)

16. from 15 keep 1001-1112 (112)

A. Database: EMBASE—up to February 2017

\#16 \#14 AND 'human'/de AND [embase]/lim NOT [medline]/lim 308

\#15 \#14 AND ‘human'/de 1489

\#14 \#5 AND \#13 2534

\#13 \#6 OR \#7 OR \#8 OR \#9 OR \#10 OR \#11 OR \#12 1688887

$\# 12$ 'sensitivity and sensibility' 982

\#11 'sensitivity' 1132406

\#10 'specificity' 719846

\#9 'pretest posttest design' 2331 


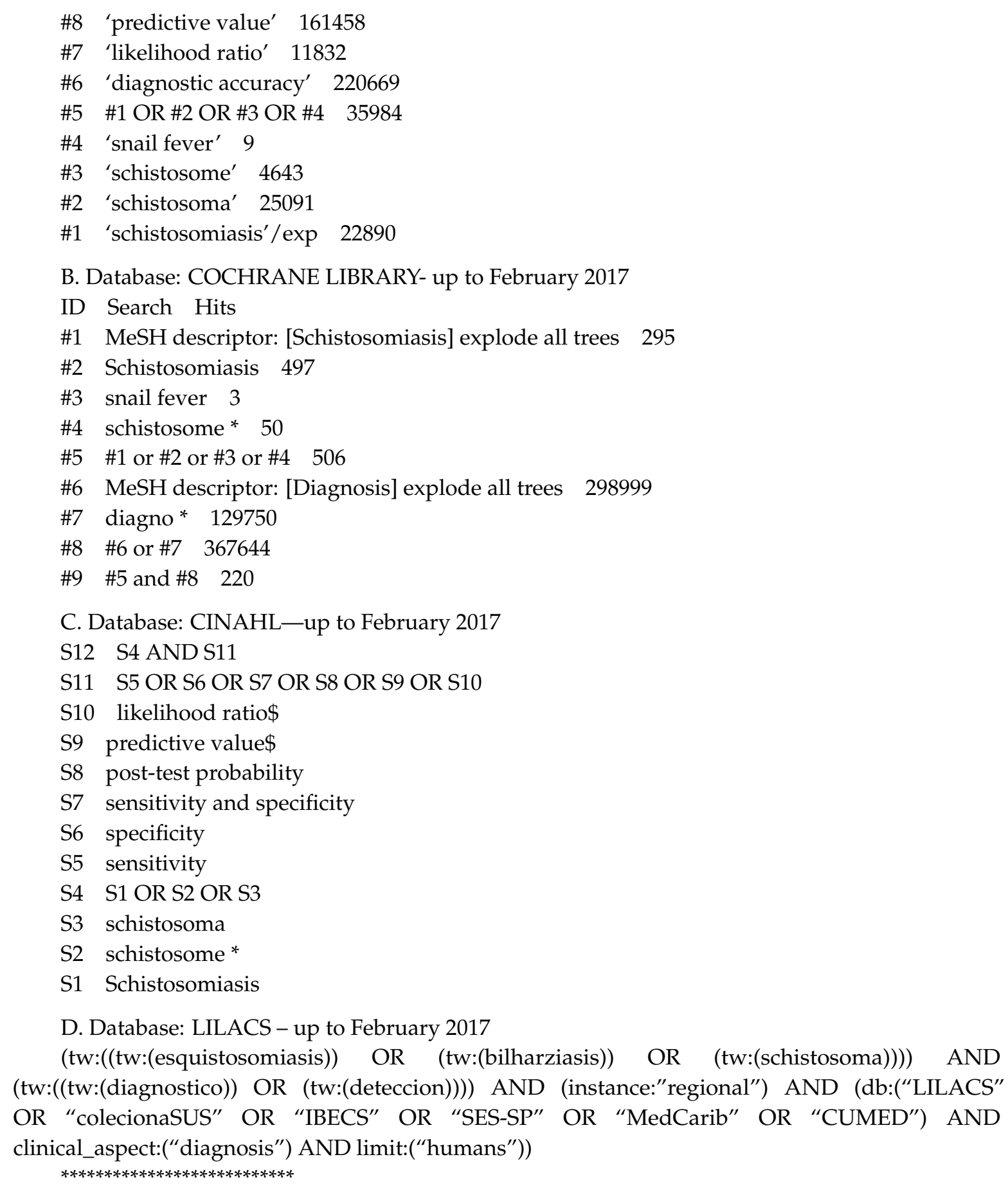

4. Update Literature strategy for primary studies on diagnostic or screening tools for strongyloidiasis

A. Database: Ovid MEDLINE(R)—1946 to February 2017

1. Strongyloidiasis/ (3403)

2. Strongyloidiasis.mp. (3747)

3. Strongyloides stercoralis/ (1098)

4. Strongyloides stercoralis.mp. (2142)

5. or/1-4 (4376)

6. exp "Sensitivity and Specificity" / (494358)

7. sensitivity.tw. (637846) 
8. $\quad$ specificity.tw. (379066)

9. ((pre-test or pretest) adj probability).tw. (1689)

10. post-test probability.tw. (438)

11. predictive value\$.tw. (84929)

12. likelihood ratio\$.tw. (11613)

13. or /6-12 (1216076)

14. 5 and $13(247)$

15. limit 14 to humans (207)

B. Database: EMBASE—up to February 2017

No. Query Results

\#14 \#12 AND [embase]/lim NOT [medline]/lim AND ‘human'/de 136

\#13 \#12 AND [embase]/lim NOT [medline]/lim 156

\#12 \#3 AND \#11 472

\#11 \#4 OR \#5 OR \#6 OR \#7 OR \#8 OR \#9 OR \#10 1686971

$\# 10$ 'diagnostic accuracy' 220414

\#9 'likelihood ratio' 11815

\#8 'predictive value' 161090

\#7 'pretest posttest design' 2315

\#6 'specificity' 719056

\#5 'sensitivity' 1131076

\#4 'sensitivity and sensibility' 981

\#3 \#1 OR \#2 5662

\#2 'strongyloides stercoralis' 3193

\#1 'strongyloidiasis'/exp 4162

C. Database: COCHRANE LIBRARY—up to February 2017

ID Search Hits

\#1 MeSH descriptor: [Strongyloidiasis] explode all trees 28

\#2 Strongyloidiasis 53

\#3 MeSH descriptor: [Strongyloides stercoralis] explode all trees 12

\#4 Strongyloides stercoralis 47

\#5 \#1 or \#2 or \#3 or \#4 72

\#6 MeSH descriptor: [Diagnosis] explode all trees 298999

\#7 diagno * 129739

\#8 \#6 or \#7 367633

\#9 \#5 and \#8 38

D. Database: CINAHL—up to February 2017

Términos de la búsqueda Opciones de búsqueda

S11 (S4 OR S5 OR S6 OR S7 OR S8 OR S9) AND (S3 AND S10)

S10 S4 OR S5 OR S6 OR S7 OR S8 OR S9

S9 likelihood ratio\$

S8 predictive value\$

S7 post-test probability

S6 sensitivity and specificity

S5 specificity

S4 sensitivity

S3 S1 OR S2

S2 strongyloides stercoralis

S1 strongyloidiasis 
E. Database: LILACS—up to February 2017

(tw:((tw:(estrongiloidiasis)) OR (tw:(Strongyloides stercoralis)))) AND (tw:((tw:(diagnostico)) OR $(\mathrm{tw}:($ deteccion) $)))$

\section{References}

1. Puthiyakunnon, S.; Boddu, S.; Li, Y.; Zhou, X.; Wang, C.; Li, J.; Chen, X. Strongyloidiasis-An insight into its global prevalence and management. PLoS Negl. Trop. Dis. 2014, 8, e3018. [CrossRef] [PubMed]

2. Riccardi, N.; Nosenzo, F.; Peraldo, F.; Sarocchi, F.; Taramasso, L.; Traverso, P.; Viscoli, C.; Di Biagio, A.; Derchi, L.E.; De Maria, A. Increasing prevalence of genitourinary schistosomiasis in Europe in the Migrant Era: Neglected no more? PLoS Negl. Trop. Dis. 2017, 11, e0005237. [CrossRef] [PubMed]

3. Murray, C.J.; Vos, T.; Lozano, R.; Naghavi, M.; Flaxman, A.D.; Michaud, C.; Ezzati, M.; Shibuya, K.; Salomon, J.A.; Abdalla, S.; et al. Disability-adjusted life years (DALYs) for 291 diseases and injuries in 21 regions, 1990-2010: A systematic analysis for the Global Burden of Disease Study 2010. Lancet 2012, 380, 2197-2223. [CrossRef]

4. King, C.H. Parasites and poverty: The case of schistosomiasis. Acta Trop. 2010, 113, 95-104. [CrossRef] [PubMed]

5. Zoni, A.C.; Catalá, L.; Ault, S.K. Schistosomiasis Prevalence and Intensity of Infection in Latin America and the Caribbean Countries, 1942-2014: A Systematic Review in the Context of a Regional Elimination Goal. PLoS Negl. Trop. Dis. 2016, 10, e0004493. [CrossRef] [PubMed]

6. Schar, F.; Trostdorf, U.; Giardina, F.; Khieu, V.; Muth, S.; Marti, H.; Vounatsou, P.; Odermatt, P. Strongyloides stercoralis: Global Distribution and Risk Factors. PLoS Negl. Trop. Dis. 2013, 7, e2288. [CrossRef] [PubMed]

7. Bisoffi, Z.; Buonfrate, D.; Montresor, A.; Requena-Mendez, A.; Munoz, J.; Krolewiecki, A.J.; Gotuzzo, E.; Mena, M.A.; Chiodini, P.L.; Anselmi, M.; et al. Strongyloides stercoralis: A plea for action. PLoS Negl. Trop. Dis. 2013, 7, e2214. [CrossRef]

8. Adenowo, A.F.; Oyinloye, B.E.; Ogunyinka, B.I.; Kappo, A.P. Impact of human schistosomiasis in sub-Saharan Africa. Braz. J. Infect. Dis. 2015, 19, 196-205. [CrossRef]

9. Hotez, P.J.; Alvarado, M.; Basanez, M.G.; Bolliger, I.; Bourne, R.; Boussinesq, M.; Brooker, S.J.; Brown, A.S.; Buckle, G.; Budke, C.M.; et al. The global burden of disease study 2010: Interpretation and implications for the neglected tropical diseases. PLoS Negl. Trop. Dis. 2014, 8, e2865. [CrossRef] [PubMed]

10. Beltrame, A.; Buonfrate, D.; Gobbi, F.; Angheben, A.; Marchese, V.; Monteiro, G.B.; Bisoffi, Z. The hidden epidemic of schistosomiasis in recent African immigrants and asylum seekers to Italy. Eur. J. Epidemiol. 2017. [CrossRef]

11. Khan, K.; Sears, J.; Chan, A.; Rashid, M.; Greenaway, C.; Stauffer, W.; Narasiah, L.; Pottie, K. Canadian Collaboration for Immigrant and Refugee Health (CCIRH). Strongyloides and Schistosoma: Evidence review for newly arriving immigrants and refugee. In The Canadian Collaboration for Immigrant and Refugee Health. Appendix 8: Intestinal Parasites; Canadian Medical Association Journal: Ottawa, ON, Canada, 2011.

12. Wilson, J.M.G.; Jungner, G.; Organization, W.H. Principles and Practice of Screening for Disease; World Health Organization: Geneva, Switzerland, 1968.

13. Colley, D.G.; Bustinduy, A.L.; Secor, W.E.; King, C.H. Human schistosomiasis. Lancet 1969, 383, $2253-2264$. [CrossRef]

14. Deniaud, F.; Rouesse, C.; Collignon, A.; Domingo, A.; Rigal, L. Failure to offer parasitology screening to vulnerable migrants in France: Epidemiology and consequences. Sante (Montrouge, France) 2010, 20, 201-208. (In French)

15. Ross, A.G.; McManus, D.P.; Farrar, J.; Hunstman, R.J.; Gray, D.J.; Li, Y.S. Neuroschistosomiasis. J. Neurol. 2012, 259, 22-32. [CrossRef] [PubMed]

16. Buonfrate, D.; Requena-Mendez, A.; Angheben, A.; Munoz, J.; Gobbi, F.; Van Den Ende, J.; Bisoffi, Z. Severe strongyloidiasis: A systematic review of case reports. BMC Infect. Dis. 2013, 13, 78. [CrossRef] [PubMed]

17. Kim, J.H.; Kim, D.S.; Yoon, Y.K.; Sohn, J.W.; Kim, M.J. Donor-Derived Strongyloidiasis Infection in Solid Organ Transplant Recipients: A Review and Pooled Analysis. Transp. Proc. 2016, 48, 2442-2449. [CrossRef] [PubMed] 
18. Berry, A.; Paris, L.; Boissier, J.; Caumes, E. Schistosomiasis Screening of Travelers to Corsica, France. Emerg. Infect. Dis. 2016, 22, 159. [CrossRef] [PubMed]

19. Requena-Mendez, A.; Chiodini, P.; Bisoffi, Z.; Buonfrate, D.; Gotuzzo, E.; Munoz, J. The laboratory diagnosis and follow up of strongyloidiasis: A systematic review. PLoS Negl. Trop. Dis. 2013, 7, e2002. [CrossRef]

20. Greaves, D.; Coggle, S.; Pollard, C.; Aliyu, S.H.; Moore, E.M. Strongyloides stercoralis infection. BMJ 2013, 347, f4610. [CrossRef]

21. Deniaud, F.; Legros, P.; Collignon, A.; Prevot, M.; Domingo, A.; Ayache, B. Targeted screening proposed in 6 migrant worker housing units in Paris in 2005: Feasibility and impact study. Sante Publique 2008, 20, 547-559. (In French) [CrossRef]

22. Chernet, A.; Kling, K.; Sydow, V.; Kuenzli, E.; Hatz, C.; Utzinger, J.; van Lieshout, L.; Marti, H.; Labhardt, N.D.; Neumayr, A. Accuracy of diagnostic tests for Schistosoma mansoni infection in asymptomatic Eritrean refugees: Serology and POC-CCA against stool microscopy. Clin. Infect. Dis. 2017. [CrossRef]

23. Weerakoon, K.G.; Gobert, G.N.; Cai, P.; McManus, D.P. Advances in the Diagnosis of Human Schistosomiasis. Clin. Microbiol. Rev. 2015, 28, 939-967. [CrossRef] [PubMed]

24. Agbata, E.N.; Padilla, P.F.; Agbata, I.N.; Armas, L.H.; Sola, I.; Pottie, K.; Alonso-Coello, P. Migrant Healthcare Guidelines: A Systematic Quality Assessment. J. Immigr. Minor. Health 2018. [CrossRef] [PubMed]

25. Eurostat. Eurostat migr_resfirst, m.r. Residence permits statistics. Available online: https:/ / ec.europa.eu/ eurostat/documents / 2995521/9333446/3-25102018-AP-EN.pdf/3fa5fa53-e076-4a5f-8bb5-a8075f639167 (accessed on 19 December 2018).

26. European Centre for Disease Prevention and Control. Monitoring implementation of the Dublin Declaration on Partnership to Fight HIV/AIDS in Europe and Central Asia: 2017 Progress Report Stockholm; European Centre for Disease Prevention and Control: Stockholm, Sweden, 2017.

27. Eurostat. Eurostat migr_asydcfsta, t. Asylum quarterly report. Available online: https://ec.europa.eu/ eurostat/statistics-explained/pdfscache/13562.pdf (accessed on 19 December 2018).

28. European Parliament. EU Migrant Crisis: Facts and Figures. 2017. Available online: http:/ /www.europarl. europa.eu/news/en/headlines/society/20170629STO78630/eu-migrant-crisis-facts-and-figures (accessed on 19 December 2018).

29. Gushulak, B.D.; MacPherson, D.W. Population mobility and health: An overview of the relationships between movement and population health. J. Travel Med. 2004, 11, 171-178. [CrossRef] [PubMed]

30. Beknazarova, M.; Whiley, H.; Ross, K. Strongyloidiasis: A disease of socioeconomic disadvantage. Int. J. Environ. Res. Public Health 2016, 13, 517. [CrossRef] [PubMed]

31. Seedat, F.; Hargreaves, S.; Nellums, L.B.; Ouyang, J.; Brown, M.; Friedland, J.S. How effective are approaches to migrant screening for infectious diseases in Europe? A systematic review. Lancet Infect. Dis. 2018, 18, e259-e271. [CrossRef]

32. Kortas, A.; Polenz, J.; von Hayek, J.; Rüdiger, S.; Rottbauer, W.; Storr, U.; Wibmer, T. Screening for infectious diseases among asylum seekers newly arrived in Germany in 2015: A systematic single-centre analysis. Public Health 2017, 153, 1-8. [CrossRef] [PubMed]

33. Aldridge, R.W.; Yates, T.A.; Zenner, D.; White, P.J.; Abubakar, I.; Hayward, A.C. Pre-entry screening programmes for tuberculosis in migrants to low-incidence countries: A systematic review and meta-analysis. Lancet Infect. Dis. 2014, 14, 1240-1249. [CrossRef]

34. Carballo, M.; Hargreaves, S.; Gudumac, I.; Maclean, E.C. Evolving migrant crisis in Europe: Implications for health systems. Lancet Glob. Health 2017, 5, e252-e253. [CrossRef]

35. Karki, T.; Napoli, C.; Riccardo, F.; Fabiani, M.; Dente, M.G.; Carballo, M.; Noori, T.; Declich, S. Screening for infectious diseases among newly arrived migrants in EU/EEA countries-varying practices but consensus on the utility of screening. Int. J. Environ. Res. Public Health 2014, 11, 11004-11014. [CrossRef]

36. Semenza, J.C.; Carrillo-Santisteve, P.; Zeller, H.; Sandgren, A.; van der Werf, M.J.; Severi, E.; Pastore Celentano, L.; Wiltshire, E.; Suk, J.E.; Dinca, I.; et al. Public Health needs of migrants, refugees and asylum seekers in Europe, 2015: Infectious disease aspects. Eur. J. Public Health 2016, 26, 372-373. [CrossRef]

37. Schunemann, H.J.; Wiercioch, W.; Brozek, J.; Etxeandia-Ikobaltzeta, I.; Mustafa, R.A.; Manja, V.; Brignardello-Petersen, R.; Neumann, I.; Falavigna, M.; Alhazzani, W.; et al. GRADE Evidence to Decision (EtD) frameworks for adoption, adaptation, and de novo development of trustworthy recommendations: GRADE-ADOLOPMENT. J. Clin. Epidemiol. 2017, 81, 101-110. [CrossRef] [PubMed] 
38. Moher, D.; Liberati, A.; Tetzlaff, J.; Altman, D.G.; Group, P. Preferred reporting items for systematic reviews and meta-analyses: The PRISMA statement. PLoS Med. 2009, 6, e1000097. [CrossRef] [PubMed]

39. Pottie, K.; Mayhew, A.D.; Morton, R.L.; Greenaway, C.; Akl, E.A.; Rahman, P.; Zenner, D.; Pareek, M.; Tugwell, P.; Welch, V.; et al. Prevention and assessment of infectious diseases among children and adult migrants arriving to the European Union/European Economic Association: A protocol for a suite of systematic reviews for public health and health systems. BMJ Open 2017, 7, e014608. [CrossRef] [PubMed]

40. Shemilt, I.; Thomas, J.; Morciano, M. A web-based tool for adjusting costs to a specific target currency and price year. Evid. Policy A J. Res. Debate Pract. 2010, 6, 51-59. [CrossRef]

41. Shea, B.J.; Grimshaw, J.M.; Wells, G.A.; Boers, M.; Andersson, N.; Hamel, C.; Porter, A.C.; Tugwell, P.; Moher, D.; Bouter, L.M. Development of AMSTAR: A measurement tool to assess the methodological quality of systematic reviews. BMC Med. Res. Methodol. 2007, 7, 10. [CrossRef] [PubMed]

42. The Newcastle-Ottawa Scale (NOS) for Assessing the Quality of Nonrandomised Studies in Meta-Analyses. Available online: http://www.ohri.ca/programs/clinical_epidemiology/oxford.asp (accessed on 19 December 2018).

43. Whiting, P.F.; Rutjes, A.W.; Westwood, M.E.; Mallett, S.; Deeks, J.J.; Reitsma, J.B.; Leeflang, M.M.; Sterne, J.A.; Bossuyt, P.M. QUADAS-2: A revised tool for the quality assessment of diagnostic accuracy studies. Ann. Intern. Med. 2011, 155, 529-536. [CrossRef] [PubMed]

44. Ochodo, E.A.; Gopalakrishna, G.; Spek, B.; Reitsma, J.B.; van Lieshout, L.; Polman, K.; Lamberton, P.; Bossuyt, P.M.M.; Leeflang, M.M.G. Circulating antigen tests and urine reagent strips for diagnosis of active schistosomiasis in endemic areas. Cochrane Database Syst. Rev. 2015. [CrossRef]

45. King, C.H.; Bertsch, D. Meta-analysis of Urine Heme Dipstick Diagnosis of Schistosoma haematobium Infection, Including Low-Prevalence and Previously-Treated Populations. PLoS Negl. Trop. Dis. 2013, 7, e2431. [CrossRef]

46. Wang, W.; Li, Y.; Li, H.; Xing, Y.; Qu, G.; Dai, J.; Liang, Y. Immunodiagnostic efficacy of detection of Schistosoma japonicum human infections in China: A meta analysis. Asian Pac. J. Trop. Med. 2012, 5, $15-23$. [CrossRef]

47. Danso-Appiah, A.; Olliaro, P.L.; Donegan, S.; Sinclair, D.; Utzinger, J. Drugs for treating Schistosoma mansoni infection. Cochrane Database Syst. Rev. 2013. [CrossRef]

48. Kramer, C.V.; Zhang, F.; Sinclair, D.; Olliaro, P.L. Drugs for treating urinary schistosomiasis. Cochrane Database Syst. Rev. 2014. [CrossRef] [PubMed]

49. Pérez del Villar, L.; Burguillo, F.J.; López-Abán, J.; Muro, A. Systematic Review and Meta-Analysis of Artemisinin Based Therapies for the Treatment and Prevention of Schistosomiasis. PLoS ONE 2012, 7, e45867. [CrossRef] [PubMed]

50. Yang, F.; Tan, X.D.; Liu, B.; Yang, C.; Ni, Z.L.; Gao, X.D.; Wang, Y. Meta-analysis of the diagnostic efficiency of the questionnaires screening for schistosomiasis. Parasitol. Res. 2015, 114, 3509-3519. [CrossRef] [PubMed]

51. Campo Polanco, L.; Gutierrez, L.A.; Cardona Arias, J. Diagnosis of Strongyloides Stercoralis infection: Meta-analysis on evaluation of conventional parasitological methods (1980-2013). Rev. Esp. Salud Publica 2014, 88, 581-600. (In French) [CrossRef] [PubMed]

52. Henriquez-Camacho, C.; Gotuzzo, E.; Echevarria, J.; White, A.C., Jr.; Terashima, A.; Samalvides, F.; Pérez-Molina, J.A.; Plana, M.N. Ivermectin versus albendazole or thiabendazole for Strongyloides stercoralis infection. Cochrane Database Syst. Rev. 2016. [CrossRef] [PubMed]

53. Danso-Appiah, A.; Minton, J.; Boamah, D.; Otchere, J.; Asmah, R.H.; Rodgers, M.; Bosompem, K.M.; Eusebi, P.; De Vlas, S.J. Accuracy of point-of-care testing for circulatory cathodic antigen in the detection of schistosome infection: Systematic review and meta-analysis. Bull. World Health Organ. 2016, 94, 522-533. [CrossRef] [PubMed]

54. Kinkel, H.F.; Dittrich, S.; Baumer, B.; Weitzel, T. Evaluation of eight serological tests for diagnosis of imported schistosomiasis. Clin. Vaccine Immunol. 2012, 19, 948-953. [CrossRef]

55. Lodh, N.; Mwansa, J.C.; Mutengo, M.M.; Shiff, C.J. Diagnosis of Schistosoma mansoni without the stool: Comparison of three diagnostic tests to detect Schistosoma [corrected] mansoni infection from filtered urine in Zambia. Am. J. Trop. Med. Hyg. 2013, 89, 46-50. [CrossRef]

56. Espirito-Santo, M.C.; Alvarado-Mora, M.V.; Dias-Neto, E.; Botelho-Lima, L.S.; Moreira, J.P.; Amorim, M.; Pinto, P.L.; Heath, A.R.; Castilho, V.L.; Goncalves, E.M.; et al. Evaluation of real-time PCR assay to detect Schistosoma mansoni infections in a low endemic setting. BMC Infect. Dis. 2014, 14, 558. [CrossRef] 
57. Espirito-Santo, M.C.; Alvarado-Mora, M.V.; Pinto, P.L.; Sanchez, M.C.; Dias-Neto, E.; Castilho, V.L.; Goncalves, E.M.; Chieffi, P.P.; Luna, E.J.; Pinho, J.R.; et al. Comparative Study of the Accuracy of Different Techniques for the Laboratory Diagnosis of Schistosomiasis Mansoni in Areas of Low Endemicity in Barra Mansa City, Rio de Janeiro State, Brazil. Biomed. Res. Int. 2015, 2015, 135689. [CrossRef]

58. da Frota, S.M.; Carneiro, T.R.; Queiroz, J.A.; Alencar, L.M.; Heukelbach, J.; Bezerra, F.S. Combination of Kato-Katz faecal examinations and ELISA to improve accuracy of diagnosis of intestinal schistosomiasis in a low-endemic setting in Brazil. Acta Trop. 2011, 120 (Suppl. 1), S138-S141. [CrossRef] [PubMed]

59. Silveira, A.M.; Costa, E.G.; Ray, D.; Suzuki, B.M.; Hsieh, M.H.; Fraga, L.A.; Caffrey, C.R. Evaluation of the CCA Immuno-Chromatographic Test to Diagnose Schistosoma mansoni in Minas Gerais State, Brazil. PLoS Negl. Trop. Dis. 2016, 10, e0004357. [CrossRef] [PubMed]

60. Espirito-Santo, M.C.; Sanchez, M.C.; Sanchez, A.R.; Alvarado-Mora, M.V.; Castilho, V.L.; Goncalves, E.M.; Luna, E.J.; Gryschek, R.C. Evaluation of the sensitivity of IgG and IgM ELISA in detecting Schistosoma mansoni infections in a low endemicity setting. Eur. J. Clin. Microbiol. Infect. Dis. 2014, 33, 2275-2284. [CrossRef] [PubMed]

61. Beltrame, A.; Guerriero, M.; Angheben, A.; Gobbi, F.; Requena-Mendez, A.; Zammarchi, L.; Formenti, F.; Perandin, F.; Buonfrate, D.; Bisoffi, Z. Accuracy of parasitological and immunological tests for the screening of human schistosomiasis in immigrants and refugees from African countries: An approach with Latent Class Analysis. PLoS Negl. Trop. Dis. 2017, 11, e0005593. [CrossRef] [PubMed]

62. Bisoffi, Z.; Buonfrate, D.; Sequi, M.; Mejia, R.; Cimino, R.O.; Krolewiecki, A.J.; Albonico, M.; Gobbo, M.; Bonafini, S.; Angheben, A.; et al. Diagnostic accuracy of five serologic tests for Strongyloides stercoralis infection. PLoS Negl. Trop. Dis. 2014, 8, e2640. [CrossRef] [PubMed]

63. Rascoe, L.N.; Price, C.; Shin, S.H.; McAuliffe, I.; Priest, J.W.; Handali, S. Development of Ss-NIE-1 recombinant antigen based assays for immunodiagnosis of strongyloidiasis. PLoS Negl. Trop. Dis. 2015, 9, e0003694. [CrossRef] [PubMed]

64. Knopp, S.; Salim, N.; Schindler, T.; Karagiannis Voules, D.A.; Rothen, J.; Lweno, O.; Mohammed, A.S.; Singo, R.; Benninghoff, M.; Nsojo, A.A.; et al. Diagnostic accuracy of Kato-Katz, FLOTAC, Baermann, and PCR methods for the detection of light-intensity hookworm and Strongyloides stercoralis infections in Tanzania. Am. J. Trop. Med. Hyg. 2014, 90, 535-545. [CrossRef] [PubMed]

65. King, C.H.; Olbrych, S.K.; Soon, M.; Singer, M.E.; Carter, J.; Colley, D.G. Utility of Repeated Praziquantel Dosing in the Treatment of Schistosomiasis in High-Risk Communities in Africa: A Systematic Review. PLoS Negl. Trop. Dis. 2011, 5, e1321. [CrossRef] [PubMed]

66. Muennig, P.; Pallin, D.; Sell, R.L.; Chan, M.-S. The Cost Effectiveness of Strategies for the Treatment of Intestinal Parasites in Immigrants. N. Engl. J. Med. 1999, 340, 773-779. [CrossRef]

67. Muennig, P.; Pallin, D.; Challah, C.; Khan, K. The cost-effectiveness of ivermectin vs. albendazole in the presumptive treatment of strongyloidiasis in immigrants to the United States. Epidemiol. Infect. 2004, 132, 1055-1063. [CrossRef]

68. Maskery, B.; Coleman, M.S.; Weinberg, M.; Zhou, W.; Rotz, L.; Klosovsky, A.; Cantey, P.T.; Fox, L.M.; Cetron, M.S.; Stauffer, W.M. Economic Analysis of the Impact of Overseas and Domestic Treatment and Screening Options for Intestinal Helminth Infection among US-Bound Refugees from Asia. PLoS Negl. Trop. Dis. 2016, 10, e0004910. [CrossRef] [PubMed]

69. Worrell, C.M.; Bartoces, M.; Karanja, D.M.; Ochola, E.A.; Matete, D.O.; Mwinzi, P.N.; Montgomery, S.P.; Secor, W.E. Cost analysis of tests for the detection of Schistosoma mansoni infection in children in western Kenya. Am. J. Trop. Med. Hyg. 2015, 92, 1233-1239. [CrossRef] [PubMed]

70. Libman, M.D.; MacLean, J.D.; Gyorkos, T.W. Screening for schistosomiasis, filariasis, and strongyloidiasis among expatriates returning from the tropics. Clin. Infect. Dis. 1993, 17, 353-359. [CrossRef] [PubMed]

71. CDC. Guidelines for Overseas Presumptive Treatment of Strongyloidiasis, Schistosomiasis, and Soil-Transmitted Helminth Infections; CDC: Atlanta, GA, USA, 2013.

72. Buonfrate, D.; Sequi, M.; Mejia, R.; Cimino, R.O.; Krolewiecki, A.J.; Albonico, M.; Degani, M.; Tais, S.; Angheben, A.; Requena-Mendez, A.; et al. Accuracy of five serologic tests for the follow up of Strongyloides stercoralis infection. PLoS Negl. Trop. Dis. 2015, 9, e0003491. [CrossRef] [PubMed] 
73. Zammarchi, L.; Bonati, M.; Strohmeyer, M.; Albonico, M.; Requena-Méndez, A.; Bisoffi, Z.; Nicoletti, A.; García, H.H.; Bartoloni, A. Screening, diagnosis and management of human cysticercosis and T. solium taeniasis: Technical recommendations by the COHEMI project study group. Trop. Med. Int. Health 2017, 2, 881-894. [CrossRef] [PubMed]

74. Jonas, D.E.; Ferrari, R.M.; Wines, R.C.; Vuong, K.T.; Cotter, A.; Harris, R.P. Evaluating evidence on intermediate outcomes: Considerations for groups making healthcare recommendations. Am. J. Prev. Med. 2018, 54, S38-S52. [CrossRef] [PubMed]

75. Atkins, D.; Best, D.; Briss, P.A.; Eccles, M.; Falck-Ytter, Y.; Flottorp, S.; Guyatt, G.H.; Harbour, R.T.; Haugh, M.C.; Henry, D. Grading quality of evidence and strength of recommendations. BMJ 2004, 328, 1490.

2018 by the authors. Licensee MDPI, Basel, Switzerland. This article is an open access article distributed under the terms and conditions of the Creative Commons Attribution (CC BY) license (http://creativecommons.org/licenses/by/4.0/). 